\title{
Energy-Efficient Resource Allocation in Multicarrier NOMA Systems with Fairness
}

\author{
Alemu Jorgi Muhammed, Student Member, IEEE, Zheng Ma, Member, IEEE, Panagiotis D. \\ Diamantoulakis, Senior Member, IEEE, Li Li, George K. Karagiannidis, Fellow, IEEE
}

\begin{abstract}
Non-orthogonal multiple access (NOMA) has attracted both academic and industrial interest since it has been considered as one of the promising 5G technologies in order to increase connectivity and spectral efficiency. In this paper, we focus on a downlink multicarrier (MC) NOMA network, where a single base station serves a set of users through multiple subchannels. The goal is to jointly optimize energy efficiency (EE) and fairness among users with respect to the subcarrier and power allocation parameters. To achieve this with acceptable complexity, a novel greedy subcarrier assignment scheme based on the worst-user first principle is proposed. Due to the fractional form of the EE expression and the existence of interference, the power allocation problem is non-convex and NP-hard. To this end, we first transform this into an equivalent subtractive form, which is then solved by using fractional programming with sequential optimization of the inter/intra-subchannel power allocation vectors. Simulation results reveal the effectiveness of the proposed scheme in terms of $\mathrm{EE}$ and fairness among users compared to baseline schemes. Finally, the proposed algorithms are of fast convergence, low complexity, and insensitive to the initial values.
\end{abstract}

Index Terms-Non-Orthogonal Multiple Access, Successive Interference Cancellation, Quality of service, Energy Efficiency, Power Allocation

\section{INTRODUCTION}

W ITH the explosive growth of the internet-of-things (IoT), and the cloud-based applications, wireless communications require a paradigm shift to support large-scale connectivity and diverse data and latency requirements. To this direction, non-orthogonal multiple access (NOMA) has

Manuscript received XXXXXX; revised XXXXXX; accepted XXXXXX. Date of publication XXXXXX; date of current version XXXXXX. This work was supported by National Natural Science Foundation of China (No. U1734209, No. 61571373, No. U1709219 ), Key International Cooperation Project of Sichuan Province (No. 2017HH0002), Marie Curie Fellowship (No. 796426 ), NSFC China-Swedish project (No. 6161101297), and 111 Project No.111-2-14. (Corresponding author: Zheng Ma).

Part of this work has been accepted to IEEE Vehicular Technology Conference, Hawaii, USA, September 2019.

Alemu Jorgi Muhammed is with the School of Information Science and Technology, Southwest Jiaotong University, Chengdu, China and also with the school of Informatics, Wollo University, Dessie, Ethiopia (e-mail: alemu_jorgi@my.swjtu.edu.cn)

Zheng $\mathrm{Ma}$ and $\mathrm{Li} \mathrm{Li}$ are with the School of Information Science and Technology, Southwest Jiaotong University, Chengdu, China and with the (e-mails: zma@home.swjtu.edu.cn, 115e08@home.swjtu.edu.cn).

P. D. Diamantoulakis and G. K. Karagiannidis are with the School of Information Science and Technology, Southwest Jiaotong University, Chengdu, China and with the Department of Electrical and Computer Engineering, Aristotle University of Thessaloniki, 54 124, Thessaloniki, Greece and with the (e-mails: padiaman@ieee.org, geokarag@auth.gr).

Color versions of one or more of the figures in this paper are available online at http://ieeexplore.ieee.org

Digital Object Identifier XX.XXXX/TCOMM.201X.XXXXXX attached great interest from both academia and industry [1], due to its superiority in gaining spectral efficiency, mass connectivity and low latency, compared to orthogonal multiple access (OMA). Even though intra-cell interference is increased, NOMA can simultaneously serve multiple users over the power domain (PD), by using the same spectrum band [2]. PD-NOMA uses superposition coding (SC) to broadcast multiple users' message signals by considering the difference of their channel gain conditions. At the receiving end, each user applies successive interference cancellation (SIC) to extract its own signal from the aggregate received signal.

The integration of NOMA in current wireless communication technology creates several challenges, due to multipath transmission, low signal strength, and intra-cell interference [1], [3]. Also, the utilization of the entire bandwidth by all users might be prohibitive in terms of complexity. To this end, NOMA can be combined with OMA schemes in order to design wireless communication schemes with practical value. For example, multicarrier NOMA (MC-NOMA) can be used [1], [2], which enables the simultaneous utilization of a subset of subcarriers from solely a subset of users. Moreover, it is useful to consider an efficient resource allocation technique, which can achieve high transmission rate, low complexity, small latency, and seamless connectivity through network coverage. Furthermore, an effective method for adaptive bandwidth and power allocation is urgently required, in order to avoid the inevitable "spectrum crunch", due to the limited bandwidth and increasing number of users.

\section{A. Related works}

Resource allocation for NOMA has been investigated in [4] and [5], where, the primary focus has been on the sum rate maximization under the total power and proportional rate constraints. Furthermore, MC-NOMA was investigated in [6] and [7]. In [6], by considering perfect channel state information (CSI) at the base station (BS), a near optimal solution for power allocation was proposed, while in [7], an efficient power allocation scheme under imperfect CSI for different quality-of-service (QoS) requirements was introduced. In the aforementioned studies, the ultimate goal was to minimize the total transmit power. Besides, joint power allocation and subcarrier assignment for NOMA has been investigated in [9]-[11]. More specifically, a suboptimal joint power and subcarrier allocation was presented in [9], for the maximization of the weighted system throughput. Furthermore, in [10], the authors investigated the optimal power allocation under 
QoS constraints in order to maximize the weighted sum rate and in [11], the authors presented theoretical insights and an algorithm for the sum rate maximization. However, these schemes maximize either the system throughput or the overall sum rate maximization, where user fairness is not considered, which is of crucial criterion in the design on NOMA networks.

Several works have been studied for resource allocation in NOMA to ensure fairness, e.g., [12]-[15]. The power allocation scheme for NOMA networks with $\alpha$-fairness consideration was studied in [12]. Moreover, the optimal power allocation based on max-min fairness for users on a single channel was investigated in [13] and [14], using statistical CSI and instantaneous CSI, respectively. The authors of [15] exploited the proportional fairness scheduling to maximize the weighted max-min fairness, where the optimal solution was only achieved for two users on a single resource block. It is notable that the aforementioned works in NOMA consider user fairness in terms of achievable rate under the maxmin optimization approach. However, no works have been considered on the max-min optimization to ensure fairness of EE among users.

Driven by the rapid growth of data traffic and wireless terminals, energy consumption of wireless networks has been rapidly increasing, and thus energy-efficient design for the next generations of wireless communication system is of paramount importance [45]. To this end, resource allocation scheme which aim to improve the EE has become an important research topic in the design of NOMA networks. For example, in [17], an energy-efficient power allocation strategy in millimeter wave massive MIMO with NOMA has been investigated. In [18], an energy-efficient transmission scheme has been studied for SISO-NOMA systems. Moreover, the joint power allocation and channel assignment for maximizing the EE in NOMA systems was considered in [19]. The same authors in [20] further extended the work in [19] proposing a joint subchannel and power optimization framework for the downlink NOMA heterogeneous network to improve the EE. However, the proposed solution focused solely on improving the overall system's EE, which result in unbalanced use of network resources.

\section{B. Motivation and contribution}

The works mentioned above [17]-[20], mainly focus on the improvements of the overall system's EE, which is described as the ratio of sum-rate and the overall energy consumption of all users. The overall EE is a significance performance metric for system design, however, the system mainly benefits from users in better channel conditions or low sever of interference and thus, improvements obtained at the cost of users in the poor channel conditions [40]. Thus, the overall EE causes unfairness among users [40], which is a challenging problem in practical MC-NOMA networks [44]. On the other hand, the EE for each individual user is a particularly useful metric, since it can provide higher performance to the weaker users, while also reducing the utilized energy [16], [33]. Thus, different from the existing works [17]-[20], in this paper, we investigate a fairness based optimization in downlink MC-NOMA systems to maximize the individual $\mathrm{EE}$ which is expressed as the ratio of the user rate to its consumed power (bits/Joule) [16], [22]. For this purpose, we choose the max-min approach to be the objective function, which apart from EE, also preserves fairness among all users in the system [40]. The max-min optimization approach can provide fairness for all users, which is particularly important in networks where some users may have stringent EE requirement.

To the best of our knowledge, the max-min optimization approach to maximize EE while ensuring fairness among users by jointly optimizing the subcarrier and power allocation in MC-NOMA network has not been considered in the open literature. Meanwhile, an energy-efficient resource allocation that considers user's fairness is of vital importance for the next-generation communication systems in order to share resources fairly while maximizing the EE. To this end, this paper investigates for the first time in existing literature the max-min optimization for energy-efficient resource allocation in downlink MC-NOMA systems aiming at improving the EE with fairness. Therefore, in this study, we focus on the most common fairness indication, the max-min EE metric [25], which aims to guarantee fairness for all users by maximizing the minimum EE in the network for the overall available subbands, which motivates the research in this treatise. Moreover, the advantages of this study over the existing works in NOMA is that it refers to MC systems, while it preserves both fairness and energy efficiency.

Furthermore, several iterative algorithms are proposed to solve the problem of EE maximization in NOMA networks, e.g., in single cell NOMA system [19], in NOMA HetNets [20] and for massive MIMO networks in [26]. Although the iterative approach has been applied to various scenarios, the network setting that we consider in this paper is very different, making the existing solutions not directly applicable. For example, if some rules of fairness requirement is strictly imposed in order to guarantee the fairness among all users, the solutions developed in [19], [20], [26] are no longer applicable. To this end, we adopt the sequential successive convex approximation (SCA) techniques to systematically address the critical issue of the inter/intra interference of users in the MC-NOMA networks to maximize users with lowest EE performance. In this setting, we are interested in maximizing the minimum individual $\mathrm{EE}$ under the power and minimum rate constraints to optimally allocate the subchannels and transmit power. Moreover, the main contributions of the study are summarized as follows:

- We propose and investigate the maximization of the minimum individual EE under the transmit power and QoS requirements to guarantee fairness among users. The optimization problem of interest is a non-convex problem and, thus, difficult to solve directly due to the fractional structure in the EE expression and the binary variable in the channel allocation indicator. We first decompose the original non-convex problem into two subproblems, namely subchannel assignment and power allocation. As a result, the original problem is solved by a twostage algorithm that involves approximation and relaxations. We also prove that the max-min EE maximization problem in MC-NOMA is NP-hard with respect to joint subcarrier and 
power allocation.

- Then, in the first step, we propose a low complexity sub-optimal subcarrier assignment scheme. This is achieved through a greedy algorithm, which incur a reduced computational complexity compared to its exhaustive-searching counterparts.

- Based on the proposed subchannel assignment algorithm, the power allocation subproblem is formulated as a nonconvex one due to the existence of the intra-group interference in NOMA networks and the fractional expression in the objective function. Then, by exploiting the property of fractional programming, the fractional form non-convex optimization is transformed into one of tractable form. Finally, we invoke the framework of SCA [34] to iteratively update the power allocation vector by solving the approximate convex problem. As a result, a low complexity inter/intra subchannel power allocation scheme is proposed, which avoids the high computational complexity of the power optimization problem involving users on the same subcarrier as well as across subcarriers. We also prove the convergence of the proposed algorithm and analyze its complexity in practical MC-NOMA networks.

- Finally, suboptimal power-subcarrier allocation policies are proposed for iteratively improving the EE. Simulations confirm that the MC-NOMA system with the proposed subcarrier assignment and power allocation lead to a considerable performance gain compared to existing works, in terms of both EE and fairness. The proposed scheme achieves near similar performance to the exhaustive-search method at significantly lower computational complexity.

\section{Structure}

The remaining part of the paper is organized as follows: Section II presents the MC-NOMA system model and problem formulation. In section III, we propose a low complexity greedy based subcarrier assignment scheme. Section IV, presents the fractional programming together with SCP approach to propose an iterative power control algorithm and suboptimal user power allocation scheme to allocate the available power on multiplexed users. Finally, the performance of the proposed method is evaluated in section $\mathrm{V}$ by computer simulation, while the paper is concluded in section VI.

\section{System Model and Problem Formulation}

In this section, we introduce the system model of the considered downlink MC-NOMA systems, while we also formulate the problem of energy-efficient optimization problem to maximize the minimum users' $\mathrm{EE}$ with both subcarrier assignment and power allocation.

\section{A. System Model}

A single-cell based downlink MC-NOMA system scenario is considered, where a BS simultaneosuly transmits information to $\mathrm{K}$ users, as illustrated in Fig.1. All transceivers are equipped with a single-antenna. Let $P_{t}$ denote the total transmit power. The total available bandwidth $B$ is equally

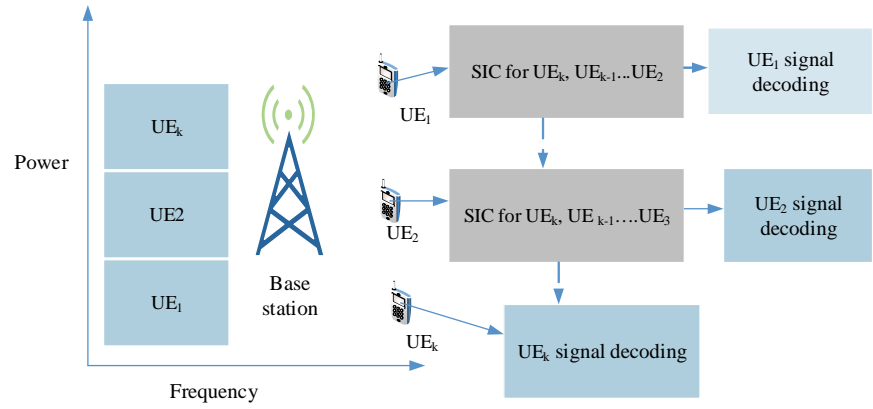

Fig. 1: Downlink NOMA for $K$ users through power domain multiplexing

divided into $N$ subcarriers, each with a bandwidth of $W=$ $\frac{B}{N}$. In this paper, the terms subchannel and subcarrier are used interchangeably. In addition, we assume that each user can occupy only $S$ subcarriers and each of the $N$ subcarriers is allocated at most $K_{n}$ users. The channel between user $k$ and the BS on subcarrier $n$ is denoted by $h_{k, n}$, and we assume that the BS has perfect knowledge of CSI. Based on the CSI of each channel, the BS assigns a subset of subchannels to the users and allocates different levels of power to them. Let $K_{n} \in\left\{K_{1}, K_{2}, \ldots, K_{N}\right\}$ be the number of users using subchannel $n=\{1,2,3, \ldots N\}$ and $\mathrm{UE}_{k, n}$ denotes user $k$ on each subchannel $n$ for $k=\left\{1,2,3, \ldots K_{n}\right\}$. Then, the corresponding transmitted signal on each subchannel $n$ is represented by

$$
x_{n}=\sum_{k=1}^{K_{n}} \sqrt{p_{k, n}} s_{k},
$$

where $s_{k}$ is the symbol of $U E_{k, n}$ and $p_{k, n}$ is the power allocated to the $k$-th user over the $n$-th subchannel (i.e., $\left.U E_{k, n}\right)$. The received signal at $\mathrm{UE}_{k, n}$ is

$$
y_{k, n}=\sqrt{p_{k, n}} h_{k, n} s_{k}+\sum_{i=1, i \neq k}^{K_{n}} \sqrt{p_{i, n}} h_{k, n} s_{i}+z_{k, n},
$$

where $h_{k, n}=g_{k, n} d_{k}^{-\gamma}$ is the channel coefficient from the BS to $\mathrm{UE}_{k, n}$ and $g_{k, n}$ is the small scale fading parameter that follows a complex Gaussian distribution, i.e., $g_{k, n} \sim C N(0,1)$, $d_{n}$ is the distance between the BS and $\mathrm{UE}_{k, n}, \gamma$ is the path loss exponent, and $z_{k, n} \sim C N\left(0, \alpha_{n}^{2}\right)$ is the additive white Gaussian noise (AWGN).

Using the main principle of power-domain NOMA, multiuser signal separation is conducted at the receiver side using the SIC approach [2]. By exploiting SIC and assuming perfect CSI, the users with better channel conditions can successfully decode the messages of the weaker users. Let $\Upsilon_{k, n}=\frac{\left|h_{k, n}\right|^{2}}{\alpha_{n}^{2}}$ denotes the channel response normalized by noise (CRNN) and consider that $K_{n}$ users are allocated on the $n$-th subchannel. Without loss of generality, the users at the $n$-th subchannel are sorted in a descending order as $\Upsilon_{1, n} \geq \cdots \geq \Upsilon_{k, n} \cdots \geq \Upsilon_{k_{n}, n}$. Thus, $\mathrm{UE}_{1, n}$ is the user which has the best channel conditions on subcarrier $n$, while $U E_{K_{n}, n}$ is the user which has the worst channel condition on the same subcarrier on channel $n$. According to the NOMA protocol [23], the BS will allocate more power to the weaker 
users to provide fairness and facilitate the SIC process, which results in $p_{1, n} \leq \cdots \leq p_{k, n} \leq \cdots \leq p_{K_{n}, n}$. Note that the first user (the user with the best channel conditions) will cancel interference from all other users, while the last user $\left(K_{n}\right)$ will see interference from all other users when decoding its own message. In general, $\mathrm{UE}_{k, n}$ is able to decode signals of $\mathrm{UE}_{i, n}$ for $i>k$ and remove them from its own signals, but treats the signals from $U E_{i, n}$ for $i<k$ as interference. Thus, the interference $\left(I_{k, n}\right)$ experienced by each user on each subcarrier with this decoding order will be [19]

$$
I_{k, n}=\sum_{i=1, i \neq k}^{K_{n}-1} p_{i, n} \Upsilon_{k, n}
$$

Hence, the received signal to the interference plus noise ratio (SINR) of the $k$-th user on subchannel $n$ is written as

$$
\operatorname{SINR}_{k, n}=\frac{P_{k, n}\left|h_{k, n}\right|^{2}}{\alpha^{2}{ }_{n}+I_{k, n}}=\frac{P_{k, n} \Upsilon_{k, n}}{1+\sum_{i=1, i \neq k}^{K_{n}-1} p_{i, n} \Upsilon_{k, n}},
$$

where $\alpha^{2}{ }_{n}=E\left\lceil\left|z_{k, n}\right|^{2}\right\rceil$ is the noise power and $\Upsilon_{k, n}=\frac{\left|h_{k, n}\right|^{2}}{\alpha_{n}^{2}}$ represents the channel response normalized by noise of the ${ }^{n} k$ th user. Thus, the data rate of $k$-th user is [14]

$$
R_{k, n}=W \log _{2}\left(1+S I N R_{k, n}\right) .
$$

Furthermore, let $P_{n}$ is the power allocated over subchannel $n$, then the subchannel power budget and BS power constraints can be expressed as

$$
\sum_{k \in K}^{K_{n}} P_{k, n}=P_{n}
$$

and

$$
\sum_{n=1}^{N} p_{n} \leq P_{t}
$$

respectively. Accordingly, as there are $K_{n}$ users on subchannel $\mathrm{n}$ and $N$ subchannels in the system, the data rate on subchannel $n$ and the total sum rate is given by

$$
R_{n}\left(P_{n}\right)=\sum_{n=1}^{K_{n}} R_{k, n}\left(P_{k, n}\right)
$$

and

$$
R=\sum_{n=1}^{N} R_{n}\left(P_{n}\right)
$$

respectively. Moreover, the overall power consumed by each user can be expressed as

$$
P_{k, n}^{\mathrm{T}}=\zeta P_{k, n}+P_{k, n}^{C},
$$

where $\zeta$ represents the inverse of the power amplifier efficiency, $P_{k, n}^{C}$ is the additional circuit power consumption of the $k$-th transmitter. Individual user's EE is defined as the ratio between the data rate and consumed power for each user [36]. This metric becomes particularly important when a balance between these two metrics is desired for all users. Thus, the EE for each user $k$ is defined as [18]

$$
E_{\eta}\left(P_{k, n}\right)=\frac{R_{k, n}\left(P_{k, n}\right)}{P_{k, n}^{\mathrm{T}}\left(P_{k, n}\right)}
$$

Moreover, in the downlink MC-NOMA, the SIC process is carrying out at the receiver side [21], [29]. This leads to high computational complexity and possibly a delay at the receiver side as the number of users grouped at the same subchannel increases. Thus, to reduce the computational complexity [19], [25], hereinafter, we consider that each user can occupy one subcarrier and only two users can be multiplexed over a particular subchannel. Thus, $K_{n}=2$, for $k=1,2 \ldots K$ and $K=2 N$. In this case, we assume that the CNRs of $\mathrm{UE}_{1, n}$ and $\mathrm{UE}_{2, n}$ are ordered as $\Upsilon_{1, n} \geq \Upsilon_{2, n}$. Then, the data rate of the strong user $\mathrm{U}_{1}$ on subchannel $n$ can be written as

$$
R_{1, n}=W \log _{2}\left(1+P_{1, n} \Upsilon_{1, n}\right),
$$

Furthermore, as the weak user $\mathrm{U}_{2}$ does not perform SIC and treats the signal from strong user as noise, then data rate of the weak user on subchannel $n$ can also be expressed as

$$
R_{2, n}=W \log _{2}\left(1+\frac{P_{2, n} \Upsilon_{2, n}}{P_{1, n} \Upsilon_{2, n}+1}\right) .
$$

\section{B. Problem Formulation}

In this section, we introduce an optimization problem for downlink MC-NOMA. Thus, given the expression for the individual $\mathrm{EE}$ for each user, the optimization problem can be formulated as

$$
\begin{array}{ll}
\max _{Q, P} \min _{k=1, \cdots, K} & E_{\eta}(Q, P)=\frac{R_{k, n}(Q, P)}{P_{k, n}^{\mathrm{T}}(Q, P)}, \\
\text { s.t. } & \mathrm{C}_{1}: \sum_{n \in N} R_{k, n} \geq R_{k}^{\mathrm{req}}, \quad \forall k \in K, \\
& \mathrm{C}_{2}: \sum_{n=1}^{N} P_{n} \leq P_{t}, \\
& \mathrm{C}_{3}: \sum_{k=1}^{K_{n}} q_{k, n} P_{k, n} \leq P_{n}, \quad \forall k \in K, \\
& \mathrm{C}_{4}: \sum_{k=1}^{K} q_{k, n} \leq K_{n}, \quad \forall n \in N, \\
& \mathrm{C}_{5}: P_{k, n} \geq 0, \quad \forall k, n, \\
& \mathrm{C}_{6}: q_{k, n} \in\{0,1\}, \quad \forall k, n,
\end{array}
$$

where the set $Q$ with elements $q_{k, n}$ and $P$ with elements $p_{k, n}$ are the subcarrier allocation policy and the power allocation strategy, respectively. Constraint $\mathrm{C}_{1}$ guarantees that all users meet their minimum QoS requirements, determined by the rate threshold $R_{k}^{\text {req }}$ for each user $k . \mathrm{C}_{2}$ and $\mathrm{C}_{3}$ are constraints for the transmission power of the BS and power budget for each subchannel $n$, respectively. $\mathrm{C}_{4}$ ensures that one subcarrier can be with at most $K_{n}$ users. $\mathrm{C}_{5}$ retains the power allocation variables to non-negative values. $\mathrm{C}_{6}$ is a subcarrier allocation variable indicator, which becomes 1 if the user $k$ is multiplexed on subcarrier $n$, and zero otherwise. Note that (14) is a nonconvex optimization problem due to the binary constraint in $\mathrm{C}_{6}$ and the existence of the interference term and fractional expression in the objective function, and also NP-hard problem [40]. In Appendix A, we will prove that the problem is NPhard .It is thus impossible to find the optimal solution within a polynomial time. 
Theorem 1: Problem (14) is an NP-hard problem (i.e., joint subcarrier and power allocation problem to maximize the EE is NP-hard problem).

Proof: See the proof in Appendix A

Once an optimization problem is shown to be NP-hard, we no longer insist on having an efficient algorithm that can find its global optimum in polynomial time [48]. Instead, we have to look at high quality approximate solutions or locally optimal solutions of the problem in polynomial time, which is more realistic in practice. Thus, it is useful to transform this into a sequence of linear programs (LPs) and develop a customized low-complexity algorithm. To make the problem tractable, we first relax $q_{k, n}$ from discrete value of 0 or 1 to continuous real numbers that range in $0 \leq q_{k, n} \leq 1, \forall(k, n) \in K \times N$ [43]. This considered as a time sharing factor for subchannel $n$ that user $k$ is assigned during one block of transmission. Now, the optimization problem in (14) can be reformulated as

$$
\begin{array}{ll}
\max _{Q, P} \min _{k=1, \cdots, K} & E_{\eta}(Q, P)=\frac{R_{k, n}(Q, P)}{P_{k, n}^{\mathrm{T}}(Q, P)} \\
\text { s.t. } & \mathrm{C}_{1}, \mathrm{C}_{2}, \mathrm{C}_{3}, \mathrm{C}_{4}, \mathrm{C}_{5}, \\
& \mathrm{C}_{6}: q_{k, n} \in[0,1], \quad \forall k, n .
\end{array}
$$

Since problem in (15) is still a fractional non-convex program, it is challenging to find an optimal solution. To this end, we next propose a two-stage algorithm, according to which the subchannel and power allocation processes are sequentially performed.

\section{ENERGY-EFFICIENT SUBCARRIER ASSIGNMENT SCHEME}

In this section, we propose a low complexity greedy based subchannel algorithm by assuming equal power allocation across the subchannels and fractional transmitted power allocation (FTPA) among multiplexed users on each subcarrier. We prefer FTPA, due to its ability to dynamically allocate power considering different channel gains among users with low complexity [19], [31]. In the FTPA scheme, the transmit power of $U E_{k}$ on subchannel $n$ is assigned based on the channel gains of all the multiplexed users on subchannel $n$, as described in [19], is given by

$$
P_{k, n}=P_{n} \frac{\left(H_{k, n}\right)^{-\sigma}}{\sum_{i=1}^{K_{n}}\left(H_{i, n}\right)^{-\sigma}},
$$

where $H$ is the channel gain of user $k$ and $i$ on subchannel $n$ and $\sigma(0 \leq \sigma \leq 1)$ is a decay factor. From (14), it can be seen that as $\sigma$ increases more power is allocated to users with lower channel gain. The procedure of our proposed suboptimal subcarrier allocation scheme for downlink MC-NOMA system is listed in Algorithm 1. The subcarrier allocation scheme aims at assigning the subcarriers to the $k$-th user, so that $\min _{k} \in$ $K, n \in N_{\left\{H_{k, n}\right\}}$ is maximized. For example, we consider a general channel quality matrix to demonstrate the operation of each algorithm when assigning users on each subcarrier. To this end, we consider a NOMA system which employs $N=4$ subcarriers to support $K=8$ users in order to allocate two users on the same subcarrier. Moreover, an OFDMA system which employs $N=4$ subcarriers to support $K=4$ users is considered since only one user is assigned for each subcarrier in OFDMA system. We initially consider an OFDMA system. The channel qualities of the 4 users with respect to 4 subcarriers are given in $(M 1)$.

$\left[\begin{array}{ccccc}\text { users } & \mathrm{U}_{1} & \mathrm{U}_{2} & \mathrm{U}_{3} & \mathrm{U}_{4} \\ \mathrm{Sc}_{1} & \underline{2.37} & 3.59 & 4.61 & 1.93 \\ \mathrm{Sc}_{2} & 1.09 & 1.90 & \mathbf{0 . 4 6} & \underline{\mathbf{0 . 0 5}} \\ \mathrm{Sc}_{3} & \mathbf{0 . 8 4} & 1.39 & \underline{3.82} & 1.96 \\ \mathrm{Sc}_{4} & 1.31 & \underline{6.60} & 5.22 & 1.65\end{array}\right]$

where the boldface shows the worst channel quality correspond to each user and the underlined numbers are channel qualities of the subcarrier assigned to users. In the case of the greedy algorithm used in [16], users one by one are allocated to subcarriers with the best channel conditions compared to the available options. As a result, user $1\left(\mathrm{U}_{1}\right)$ chooses best subcarrier from available four options. So, $\mathrm{U}_{1}$ selects the 1-st $\left(\mathrm{Sc}_{1}\right)$ subcarrier. Next, user $2\left(\mathrm{U}_{2}\right)$ selects the best subcarrier from the remaining three which is subcarrier $4\left(\mathrm{Sc}_{4}\right)$. Furthermore, user $3\left(\mathrm{U}_{3}\right)$ is assigned to subcarrier $3\left(\mathrm{Sc}_{3}\right)$. Under the lack of any other option, the subcarrier with the worst channel quality is assigned to user 4 , i.e., subcarrier $2\left(\mathrm{Sc}_{2}\right)$. Therefore, the allocated subcarriers to the four users by this algorithm are given by $\mathrm{Sc}_{1}=\left\{\mathrm{U}_{1}\right\}, \mathrm{Sc}_{2}=\left\{\mathrm{U}_{4}\right\}, \mathrm{Sc}_{3}=\left\{\mathrm{U}_{3}\right\}$ and $\mathrm{Sc}_{4}=\left\{\mathrm{U}_{2}\right\}$. Accordingly, according to this algorithm, $\mathrm{Sc}_{2}$ is assigned to $\mathrm{U}_{4}$ which has the poorest channel quality 0.05 . Therefore, one of the disadvantages of a greedy-based algorithm used by [16] is that users at the latter stage are left with limited option. Specifically, as it becomes apparent from the example, at the final stage the 2-nd subcarrier is selected to be assigned to $\mathrm{U}_{4}$, even though the corresponding channel quality of 0.05 is the worst of all. Consequently, the achievable performance will be governed by this worst subcarrier channel quality. That is $\min _{k} \in K, n \in N\left\{h_{k, n}\right\}=0.05$.

Another important subcarrier allocation algorithm used by [19] is the suboptimal matching for subchannel assignment (SOMSA) algorithm. The main idea of this algorithm is that each user sends a matching request to its most preferred subchannel. However, this subchannel has the permission to accept the user request if this results to the highest $\mathrm{EE}$, otherwise, the request will be rejected. Thus, the algorithm gives priority to users having the best channel qualities. The operation of this algorithm is demonstrated in detail by using the example in (M2). To begin with, subchannels are ordered in decreasing order of their channel gains as $\left\{\mathrm{Sc}_{4}, \mathrm{Sc}_{2}, \mathrm{Sc}_{1}, \mathrm{Sc}_{3}\right\}$ based on their best channel qualities, forming the matrix shown below:

$$
\left[\begin{array}{ccccccccc}
\text { users } & \mathrm{U}_{1} & \mathrm{U}_{2} & \mathrm{U}_{3} & \mathrm{U}_{4} & \mathrm{U}_{5} & \mathrm{U}_{6} & \mathrm{U}_{7} & \mathrm{U}_{8} \\
\mathrm{Sc}_{4} & 1.31 & \underline{6.60} & \underline{5.22} & 1.65 & 2.12 & \mathbf{0 . 5 9} & 1.02 & \mathbf{0 . 0 6} \\
\mathrm{Sc}_{2} & 1.09 & 1.90 & \mathbf{0 . 4 6} & \mathbf{0 . 0 5} & \underline{4.72} & 3.64 & \underline{4.70} & 2.37 \\
\mathrm{Sc}_{1} & \underline{2.37} & 3.59 & 4.61 & 1.93 & 1.73 & \underline{4.34} & 1.09 & 2.72 \\
\mathrm{Sc}_{3} & \mathbf{0 . 8 4} & 1.39 & 3.82 & \underline{1.96} & 1.98 & 2.47 & 1.68 & \underline{1.38}
\end{array}\right]
$$

According to (M2), the allocated subcarriers to the eight users by SOMSA algorithm are given by $\mathrm{Sc}_{1}=\left\{\mathrm{U}_{1}, \mathrm{U}_{6}\right\}$ , $\mathrm{Sc}_{2}=\left\{\mathrm{U}_{5}, \mathrm{U}_{7}\right\}, \mathrm{Sc}_{3}=\left\{\mathrm{U}_{4}, \mathrm{U}_{8}\right\}$ and $\mathrm{Sc}_{4}=\left\{\mathrm{U}_{2}, \mathrm{U}_{3}\right\}$. The worst channel quality of the allocated subcarrier in this case become $\min _{k} \in K, n \in N_{\left\{h_{k, n}\right\}}=1.38$, which shows 
significant improvement compared to greedy algorithm in [16]. Even though SOMSA is capable of achieving better allocation results compared to [16], at the last stage user $8\left(\mathrm{U}_{8}\right)$ is forced to select 1.38 value. In NOMA systems where the number of users are more than the number of subcarriers and more users are assigned to the same subcarrier, to achieve a better performance, subcarrier allocation in user oriented approach is more preferable, since it helps to avoid the assignment of subcarriers with poor channel quality [8]. Inspired by this observation, in this paper, we introduce the worst-case user first subcarrier allocation (WCUFSA) algorithm. The WCUFSA algorithm is a greedy based algorithm that allows the users with the worst channel quality to select their desired subcarrier first. To this end, users are arranged in ascending order with respect to the worst channel qualities of all users, as given in (M3). Then, the algorithm first finds the worst channel qualities of the unassigned users and then assigns the best subcarrier to the user with the poorest channel value.

$$
\left[\begin{array}{ccccccccc}
\text { users } & \mathrm{U}_{4} & \mathrm{U}_{8} & \mathrm{U}_{3} & \mathrm{U}_{6} & \mathrm{U}_{1} & \mathrm{U}_{7} & \mathrm{U}_{2} & \mathrm{U}_{5} \\
\mathrm{Sc}_{1} & 1.93 & \underline{2.72} & 4.61 & \underline{4.34} & 2.73 & 1.09 & 3.59 & 1.73 \\
\mathrm{Sc}_{2} & \mathbf{0 . 0 5} & 2.37 & \mathbf{0 . 4 6} & 3.64 & 1.09 & \underline{4.70} & \underline{1.90} & 4.72 \\
\mathrm{Sc}_{3} & \underline{1.96} & 1.38 & 3.82 & 2.47 & \mathbf{0 . 8 4} & 1.68 & 1.39 & \underline{1.98} \\
\mathrm{Sc}_{4} & 1.65 & \mathbf{0 . 0 6} & \underline{5.22} & \mathbf{0 . 5 9} & \underline{1.31} & 1.02 & 6.60 & 2.12
\end{array}\right]
$$

As shown in the considered example in (M3), $\mathrm{U}_{4}$ has the worst channel quality at 2-nd subchannel with channel gain value of 0.05 . As a result, it is the first user to select the subcarrier with the best channel quality among the available four subcarriers, which corresponds to the value 1.96. Thus, in the first column, which corresponds to the 4-th user, $\mathrm{Sc}_{3}$ has the best channel quality. Likewise, other assignments are treated in similar manner using the algorithm iteratively till all subcarriers are assigned to all users (i.e., two users per subcarrier bases). Finally, the set of allocated subcarriers becomes $\mathrm{Sc}_{1}=\left\{\mathrm{U}_{6}, \mathrm{U}_{8}\right\}, \quad \mathrm{Sc}_{2}=\left\{\mathrm{U}_{2}, \mathrm{U}_{7}\right\}, \quad \mathrm{Sc}_{3}=\left\{\mathrm{U}_{4}, \mathrm{U}_{5}\right\}$, and $\mathrm{Sc}_{4}=\left\{\mathrm{U}_{1}, \mathrm{U}_{3}\right\}$. The gain of the weakest channel utilized for transmission when WCUFSA is used becomes $\min _{k} \in K, \quad n \in N\left\{h_{k, n}\right\}=1.98$. It is clear that WCUFSA is capable of yielding the highest achievable performance in assigning better channel quality to assign a subcarrier to users, compared to the greedy algorithm and SOMSA algorithm, demonstrated in (M1) and (M2), respectively. Therefore, WCUFSA algorithm successfully avoids the assignment of channel with low channel quality even in the last stage. As a summary, the WCUFSA subcarrier allocation scheme is presented in Algorithm 1.

\section{ENERgy-EFficient Power AlLOCATION fOR NOMA SYSTEM}

In this section, we focus on power allocation optimization with the aim to further improve the EE of the NOMA network and guarantee the maximum fairness for NOMA users. The performance of NOMA depends on the selection of the user set over a particular subchannel and allocation of power to the multiplexed users on the subchannel [3], [30]. We assume that the users are assigned to different subchannels by using the subcarrier assignment algorithm, proposed in the previous

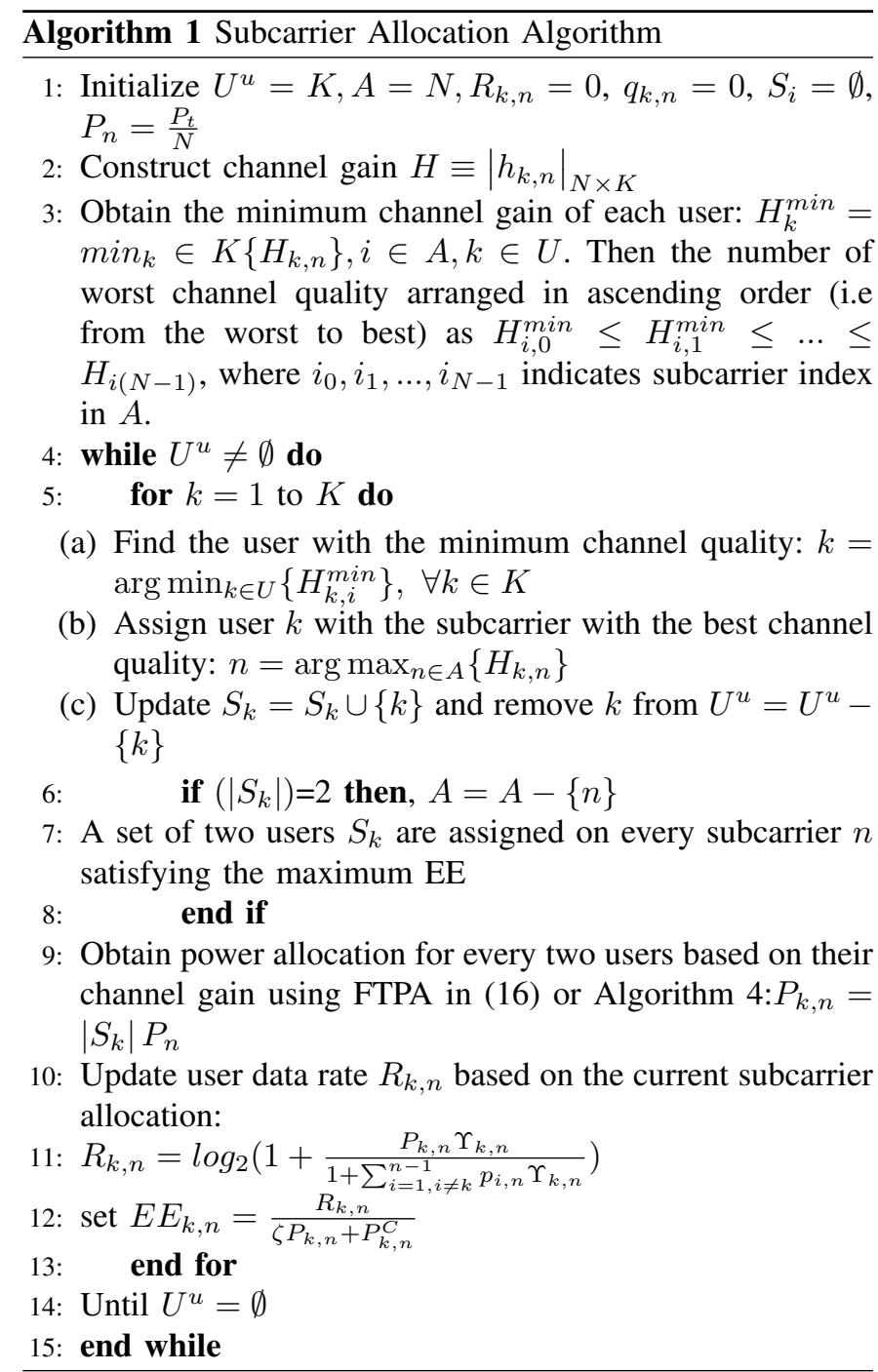

section. The resulting optimization problem can be expressed as

$$
\begin{array}{ll}
\max _{P} \min _{k=1, \cdots, K} & E_{\eta}(Q, P)=\frac{R_{k, n}(Q, P)}{P_{k, n}^{\mathrm{T}}(Q, P)} \\
\text { s.t. } & \mathrm{C}_{1}: \sum_{n \in N} R_{k, n} \geq R_{k}^{\text {req }}, \quad \forall k \in K, \\
& \mathrm{C}_{2}: \sum_{n=1}^{N} P_{n} \leq P_{t}, \\
& \mathrm{C}_{3}: \sum_{k=1}^{K_{n}} q_{k, n} P_{k, n} \leq P_{n}, \quad \forall k \in K, \\
& \mathrm{C}_{5}: P_{k, n} \geq 0, \quad \forall k, n,
\end{array}
$$

The optimization problem in (17) is still non-convex due to the fact that the objective function is the ratio of two real-value functions [16], [32], [33]. Thus, in order to obtain an optimal solution, an exhaustive search is required which is generally computationally infeasible. In order to efficiently solve (17), we transform this into the substractive form, which is more tractable. Thus, we need to introduce the following problem transformation. 


\section{A. Problem Transformation and Iterative Algorithm Design}

Since the objective function in (17) is not concave, the fractional programming tool fails to maximize the EE globally [36]. Thus, the standard convex optimization algorithm is not guaranteed to solve (17), and specific algorithms are required. As a result, we first transform (17) into its equivalent more tractable subtractive form. Without loss of generality, we assume that $R_{k, n}(Q, P)>0$ and $P_{k, n}^{T}(Q, P)>0$. For the sake of simplicity, we define $D$ as a set of feasible solutions of the optimization in (14) and $\{P, Q\} \in D$. Let $\eta^{*}$ and $P^{*}$ denote the maximum EE and optimal solution of power allocation, respectively. Thus, we define the maximum EE $\eta^{*}$ of (17) as

$$
\begin{aligned}
\eta^{*}= & \max _{P} \min _{k=1 \ldots, K} \frac{R_{k, n}(Q, P)}{P_{k, n}^{T}(Q, P)} \\
= & \min _{k} \frac{R_{k, n}\left(Q^{*}, P^{*}\right)}{P_{k, n}^{T}\left(Q^{*}, P^{*}\right)}
\end{aligned}
$$

where $(\cdot)^{*}$ denotes optimality. Based on (18), we present the following essential theorem.

Theorem 2: A vector $P^{*} \in D$ solves (17) if and only if [36], [37]

$$
\begin{aligned}
& \max _{P \in D} \min _{k=1 \ldots K}\left\{R_{k, n}(Q, P)-\eta^{*} P_{k, n}^{T}(Q, P)\right\} \\
= & \min _{k=1 \ldots K}\left\{R_{k, n}\left(Q, P^{*}\right)-\eta^{*} P_{k, n}^{T}\left(Q, P^{*}\right)\right\}=0 .
\end{aligned}
$$

Proof: See in appendix $B$

Theorem 2 reveals for an optimization problem whose objective function in fractional form can be solved by its equivalent subtractive form, i.e., we can solve (17) via (19) equivalently. Thus, the optimal solution of the auxiliary problem (19) is also the optimal solution of (17) [36], [37]. To explain in another way, solving (17) is equivalent to finding $\eta^{*}$. Let $F(\eta)$ is the optimum objective value of (17). Thus, solving (17) is essentially equivalent to finding $\eta=\eta^{*}$ with $F(\eta)=0$. Moreover, the function $F(\eta)$ is strictly decreasing in $\eta$ [36], [37]. Thus, with a given reasonable range, there is an optimal minimum EE $\eta^{*}$, satisfying $F\left(\eta^{*}\right)=0$. In addition, $F(\eta)$ is negative for $\eta \rightarrow+\infty$ and positive for $\eta \rightarrow-\infty$. Thus, the bisection iterative algorithm can be employed to determine $\eta$ since the monotonicity of $F(\eta)$ and the opposite signs at the two sides of $\eta^{*}$. To this end, the $\eta$ will reach its optimal solution when $F\left(\eta^{*}\right)=0$ and the solution for $P^{*}$ is achieved by addressing the auxiliary problem of (19) at the given minimum EE. The iterative algorithm based on the bisection method is summarized as Algorithm 2. Given a tolerance, Algorithm 2 can be used for solving the optimization problem (17) through the auxiliary problem of (19). The fundamental mathematical principle underlying the bisection method is the intermediate value theorem.

Theorem 3: Let $F$ be a continuous function on the interval $\left[\eta_{\min }, \eta_{\max }\right]$ and $F\left(\eta_{\min }\right) \cdot F\left(\eta_{\max }\right)$ are nonzero of opposite sign. Then, the optimal solution $\eta^{*}$ for $F$ is found in the interval $\left[\eta_{\min }, \eta_{\max }\right]$, which shows convergence to its solution.

\section{Proof: Refer to Appendix $C$ for the proof of convergence}

Therefore, the solution for the transmit power $P^{*}$ can be achieved by addressing the optimization problem of (20), which need to be solved at line 6 of Algorithm 2 for a given

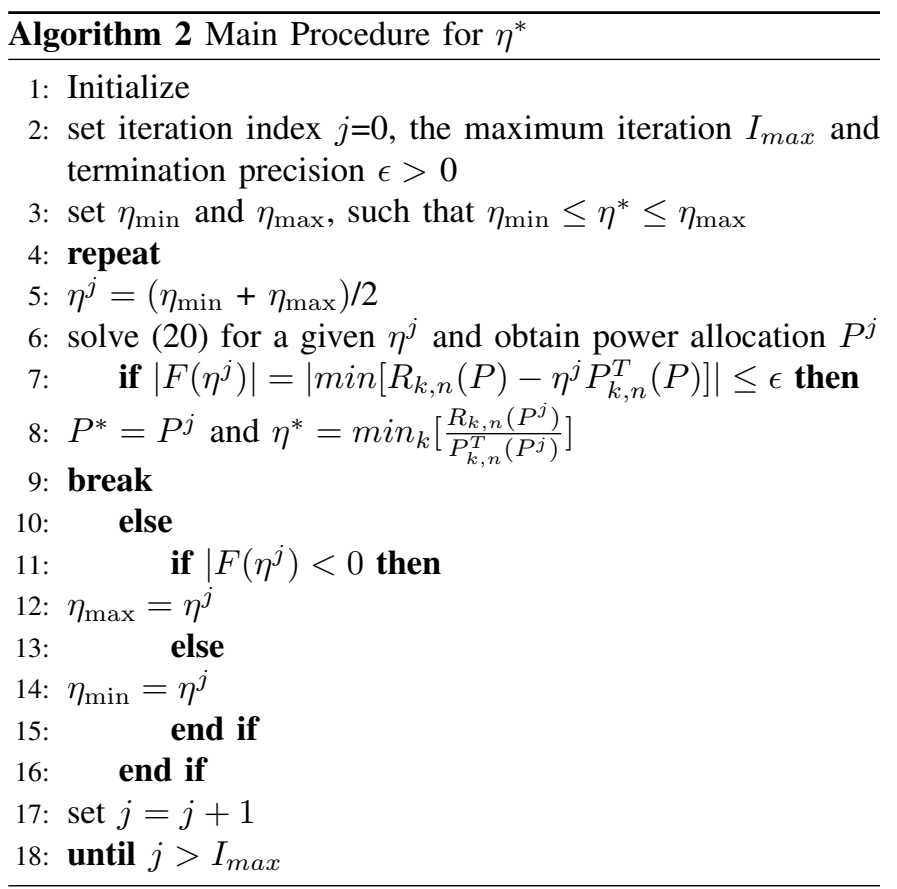

$\eta^{j}$. Thus, hereinafter, we focus on the following objective function:

$$
\begin{array}{ll}
\max _{P} \min _{k=1, \cdots, K} & \left\{R_{k, n}(Q, P)-\eta P_{k, n}^{T}(Q, P)\right\} \\
\text { s.t. } & \mathrm{C}_{1}, \mathrm{C}_{2}, \mathrm{C}_{3}, \mathrm{C}_{5} .
\end{array}
$$

The power optimization problem in (20) involves a twolevel of power allocation. The power allocation among different subchannels and the power allocation to the grouped users at the same subchannel $n$. Thus, we introduce a twolevel inter/intra-subchannel power allocation algorithm that allocates the available power among subchannels, as well as between users on the same subchannel. To provide an efficient solution to the problem, we first optimize the power allocation between subchannels. Therefore, objective function of (20) can be reformulated as

$$
\begin{array}{ll}
\max _{P_{n}} \min _{k=1, \cdots, K} & \left\{R_{k, n}(Q, P)-\eta P_{k, n}^{T}(Q, P)\right\} \\
\text { s.t. } & \mathrm{C}_{1}: \sum_{n \in N} R_{k, n}>=R_{k}^{\mathrm{req}}, \quad \forall k \in K, \\
& \mathrm{C}_{2}: \sum_{n=1}^{N} P_{n} \leq P_{t}, \\
& \mathrm{C}_{7}: P_{n} \geq 0, \quad \forall n \in N .
\end{array}
$$

Then, given the power allocation among different subchannels, we further optimize the power allocation for the two users grouped at subchannel $n$. This leads to the following optimization problem:

$$
\begin{array}{ll}
\max _{P_{k, n}} \min _{k=1, \cdots, K} & \left\{R_{k, n}(Q, P)-\eta P_{k n,}^{T}(Q, P)\right\} \\
\text { s.t. } & \mathrm{C}_{1}: \sum_{n \in N} R_{k, n}>=R_{k}^{\mathrm{req}}, \quad \forall k \in K, \\
& \mathrm{C}_{3}: \sum_{k=1}^{K_{n}} q_{k, n} P_{k, n} \leq P_{n}, \quad \forall k \in K, \\
& \mathrm{C}_{5}: P_{k, n} \geq 0, \quad \forall k \in K .
\end{array}
$$


Considering the fractional nature of the EE, the main mathematical tool for solving (21) is fractional programming [28], [36]. This principle holds when the numerator and denominator of the EE optimization problem are concave and convex respectively over convex constraint sets [36]. However, the optimization problem that needs to be solved in (21) is nonconvex with respect to the transmit power $P_{k, n}$ due to the terms of multiuser interference. Hence, we invoke the framework of SCA [34] to iteratively update the power allocation vector by solving the approximate convex problem.

\section{B. Sequential convex programming $(S C P)$ for $P^{*}$}

In this subsection, we propose an SCP optimal approach to obtain an energy-efficient power allocation scheme by iteratively solving the given problem. The proposed iterative power allocation scheme for this paper is named as nonorthogonal multiple access-sequential convex programming (NOMA-SCP). The basic idea of SCP is to approximate a nonconvex problem by a sequence of convex problems iteratively [34]. In each iteration, all non-convex constraints are replaced by their inner convex approximations [36]. Due to the nonconvexity of problem (20), it is hard to solve it directly with polynomial time complexity. To this end, the objective function in (21) can be rearranged into a difference of two concave function with respect to $P_{k}$ as

$$
R_{k, n}(P)-\eta P_{k, n}^{T}(P)=f_{k}(P)-g_{k}(P)
$$

where,

$$
\begin{gathered}
f_{k}(P)=\log _{2} \sum_{i=1}^{N} W\left(1+P_{k, n} \Upsilon_{k, n}\right)-\eta_{k} P_{k}(P) \\
g_{k}(P)=\log _{2} \sum_{i=1, i \neq k}^{N}\left(P_{i, n} \Upsilon_{k, n}+\alpha_{k, n}^{2}\right)
\end{gathered}
$$

Now, we can equivalently rewrite (20) as

$$
\begin{array}{ll}
\underset{P}{\max } \min _{k} & \left\{f_{k}(P)-g_{k}(P)\right\} \\
\text { s.t. } & \mathrm{C}_{1}, \mathrm{C}_{2}, \mathrm{C}_{7} .
\end{array}
$$

It is noted that the objective function in (26) is not smooth at each iteration of different minimum of $f_{k}(P)-g_{k}(P)$. Thus, we introduce a new variable $\mathcal{R}$ to the optimization problem (26) to transform into a smooth optimization problem. Thus, (26) can be equivalently formulated as

$$
\begin{array}{ll}
\underset{P_{n}, \mathcal{R}}{\max } & \mathcal{R} \\
\text { s.t. } & \mathrm{C}_{1}, \mathrm{C}_{2}, \mathrm{C}_{7} \\
& \mathrm{C}_{8}:\left\{f_{k}(P)-g_{k}(P)\right\} \geq \mathcal{R}, \forall k .
\end{array}
$$

It is noted that constraint $\mathrm{C}_{8}$ in (27) is the difference of two concave functions which can be effectively solved by SCP [35]. At step $t$ we can get an iterative power allocation $p^{t}$. Thus, we approximate $g_{k}(P)$ by first-order Taylor expansion at $p^{t}$, i.e.,

$$
g_{k}\left(P^{t}\right)+\nabla g_{k}^{T}\left(P^{t}\right)\left(P-P^{t}\right)
$$

where $\nabla g_{k}(P)$ is the gradient of $g_{k}(P)$ at $P$ and is given by

$$
\nabla g_{k}(P)=\frac{m_{k}}{\sum_{i=1, i \neq k} P_{i, k} \Upsilon_{k, n}+\alpha_{k, n}^{2}} .
$$

In (29) $m_{k}$ is a $K$ dimensional column vector with $m_{k}(k)=0$ and $m_{k}(i)=\frac{g_{k, i}}{\ln 2}, k \neq i$. Moreover, the minimum data rate constraint $C_{1}$ can be equivalently written as

$$
C_{1}^{\prime}: P_{k, n} \Upsilon_{k, n}+\left(1-2^{R_{k}^{\mathrm{req}} / W}\right)\left(\sum_{i=1, i \neq k}^{n-1} P_{i, n} \Upsilon_{k, n}+\alpha_{k, n}^{2}\right) \geq 0
$$

Combining (28) and (27), we can rewrite (27) as

$$
\begin{array}{ll}
\max _{P_{n}, \mathcal{R}} & \mathcal{R} \\
\text { s.t. } & \mathrm{C}^{\prime}{ }_{1}, \mathrm{C}_{2}, \mathrm{C}_{7} \\
& \mathrm{C}_{8}: f_{k}(P)-\left[g_{k}\left(P^{t}\right)+\nabla g_{k}^{T}\left(P^{t}\right)\left(P-P^{t}\right)\right] \geq \mathcal{R} .
\end{array}
$$

After this transformation, (31) is a smooth and standard convex approximation of (20). The local optimal transmit power can be efficiently calculated by solving (31). The algorithm iteratively solves the convex optimization problem in (31). We show the detailed power control algorithm in Algorithm 3.

Theorem 4: (a) The efficient iterative algorithm always converges, and (b) with any feasible initial values, the optimal transmit power converges to a stationary point of (31), i.e., (20).

Proof: See Appendix D.

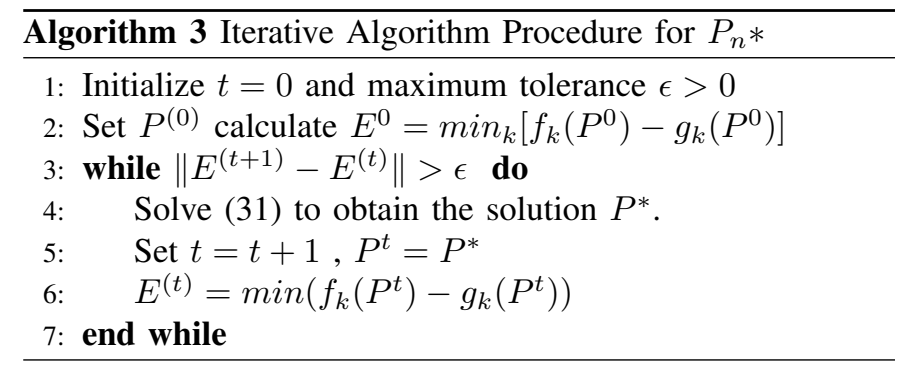

Once the power, $P_{n}$, for each subchannel $n$ is determined, the next step is to allocate power between multiplexed users on the same subchannel based on users' channel gain. According to the optimization in (22), both the strong and weak users have the same minimum data rate requirement. Users' signals will be multiplexed together using assigned powers and transmitted to users so that the total transmitted power per subchannel not to exceed from the allocated power budget, $P_{n}$. Furthermore, the transmit power of the weaker channel gain user must be higher than that of the strong channel gain user [2]. Consequently, an important conclusion about the transmission of power for the strong channel gain user in a NOMA can be drawn from [39]. In [39], the maximum power allocation to the strong channel gain user in downlink NOMA must be smaller than $\frac{P_{n}}{2^{m-1}}$, where $m$ is the number of users grouped at the same subchannel and $P_{n}$ is the power budget for each subchannel $n$ [39]. Furthermore, according to constraint $\mathrm{C}_{5}$ in (22), we have $P_{k, n} \geq 0, k \in\{1,2\}, \forall n \in N$. Thus, the power allocated to the strong channel gain user can efficiently exploit in between 0 and $\frac{P_{n}}{2^{m-1}}$. Based on our analysis, we can apply an efficient bisection search method to realize the 
suboptimal solution of power allocation for users grouped at the same subcarrier, as given in Algorithm 4.

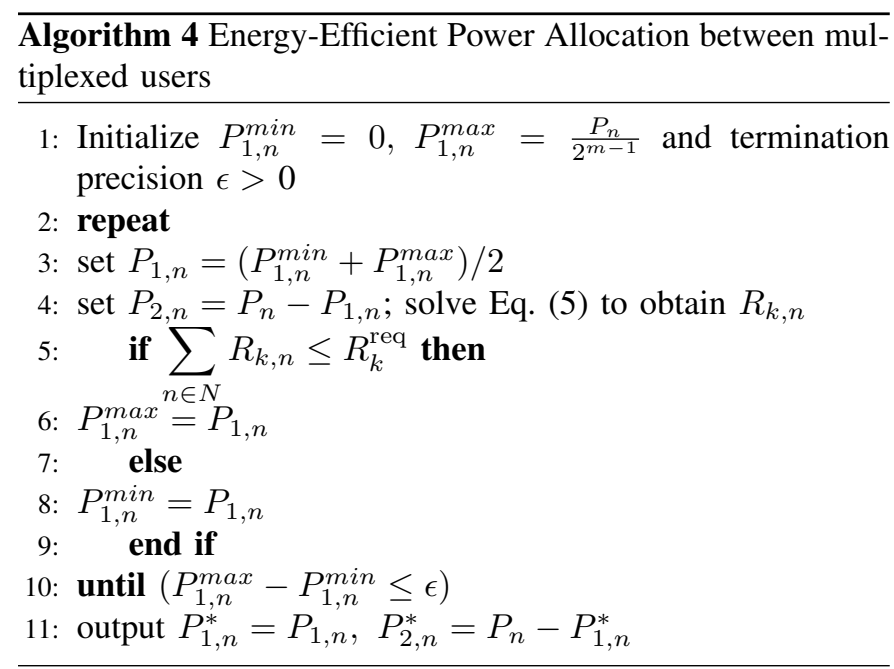

\section{Computational Complexity Analysis}

In order to get some insights for the computational complexity of the proposed algorithm, we first recall the optimal subcarrier assignment scheme which can be achieved through exhaustive search. Let us recall the $K$ users and $N$ subcarriers (i.e., $K=2 N$ ) scenario, we need to search $\frac{(2 N) !}{2^{N}}$ combinations. Thus, the complexity of the exhaustive search becomes $\mathcal{O}\left(\frac{(2 N) !}{2^{N}}\right)$ [19]. In the proposed greedy algorithm, the complexity comes from the sorting and assignment phases. In the sorting phase, the algorithm finds the minimum channel quality of $K$ users and sorts them from the lower to higher value, which requires $(K(K-1) / 2)$ operations. Furthermore, the algorithm starts from users with the worst channel quality and assigns the subcarrier with the highest channel gain, which requires $(2 K \ln K)$ operations. Therefore, the proposed subcarrier assignment algorithm requires $(K(K-1) / 2+2 K \ln K)$ operations, yielding the complexity of $\mathcal{O}\left(K^{2}\right)$. Let $L_{1}$ iterations are required to guarantee the error tolerance, $\epsilon$, for the bisection method. Also, let $L_{2}$ denotes the number of iterations required for the power allocation algorithms to converge. Thus, the total complexity of the propose schemes is therefore $\mathcal{O}\left(K^{2}+L_{1} L_{2} K N\right)$, which shows lower computational complexity compared even with the optimal subcarrier assignment algorithm alone. Thus, the proposed scheme can be implemented in polynomial time.

\section{Simulation Results}

In this part, we present simulation results to evaluate the performance of the proposed schemes, especially in comparison with the baseline schemes in [19] and [16]. We consider a single BS located in the cell center and users are uniformly distributed inside a circular ring with a radius of $300 \mathrm{~m}$. We set the value of path loss exponent $\gamma$ as 2 [25]. The minimum distance from users to $\mathrm{BS}$ is limited $50 \mathrm{~m}$. The bandwidth of the system is set as $5 \mathrm{MHz}$. As it has already been mentioned, the considered NOMA network system, two

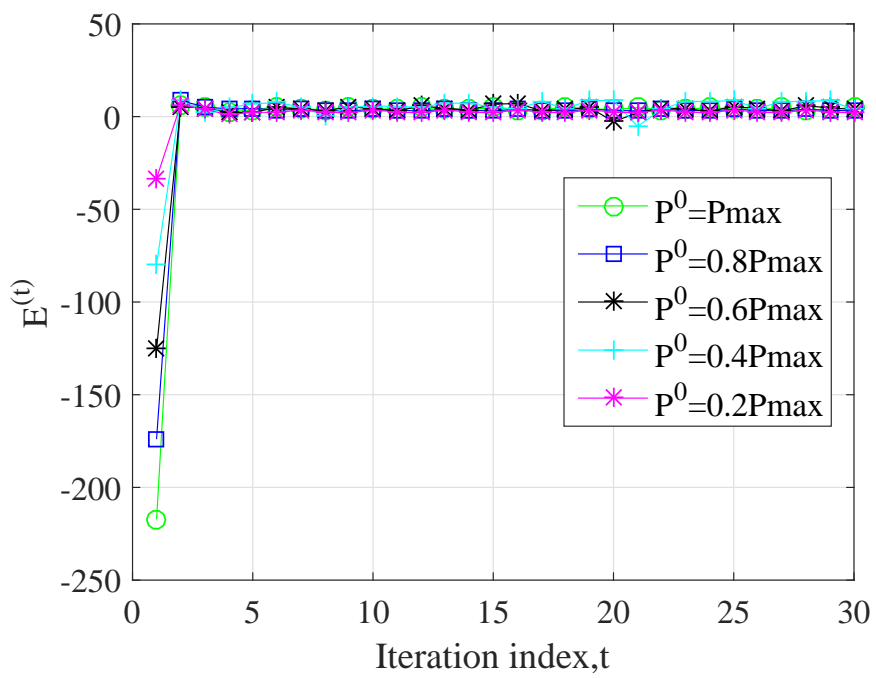

Fig. 2: The convergence of the iterative power allocation algorithm with $\eta^{j}=5$ Mbits/joule

users are assigned per subcarrier to reduce the complexity of SIC. In the simulation, we set BS peak power $P=12 W$, and circuit power consumption $\mathrm{Pc}=1 W$ [19], and $\alpha_{n}^{2}=\frac{B * N_{0}}{N}$, where $N_{0}=-174 \mathrm{dBm} / \mathrm{Hz}$ is the $\mathrm{AWGN}$ power spectral density. For simplicity, we consider each user has the same weighted bandwidth $\frac{B}{N}$. The performance of the proposed subcarrier assignment (WCUFSA) is compared to suboptimal matching for subchannel assignment algorithm in NOMA (SOMSA) [19] and OFDMA [16]. Regarding the power allocation, the performance of the proposed NOMA-SCP scheme is compared with differential convex programming (NOMA-DC) [19] and OFDMA system as well as NOMA with equal power allocation (NOMA-EQ) used in our proposed subcarrier assignment scheme. Moreover, the proposed user power allocation algorithm (UPA) for users grouped at the same subcarrier is also compared with NOMA-DC-DC [19] and FTPA (fractional transmitted power allocation), which is widely used in NOMA and OFDMA [31].

We first evaluate the feasibility and effectiveness of the proposed algorithms. Fig. 2 and Fig. 3 show the convergence behavior of the efficient iterative power allocation Algorithm and the bisection method for $\mathrm{EE}$ (i.e., $\eta^{*}$ ), respectively. It is noted that both Algorithms converge fast to reach their solution set with different initial transmit power values (i.e. $P^{0}$ ). Moreover, the Algorithms reach the solution point within a few iterations. Thus, it is proved that the proposed algorithms can reach to the solution set without being affected by the initial guess power setting. Hence, we can conclude that the proposed algorithms are of high practical value.

In Fig. 4, we compare the proposed subcarrier assignment algorithm (WCUFSA) with SOMSA and OFDMA schemes to evaluate the EE performance for n-th subcarrier as well as the overall EE performance of the whole network. $N$ in the figure denotes the $n$-th subcarrier. As can be seen in all schemes, they improve the network's EE at the cost of individual $\mathrm{EE}$ for the user with the worst channel conditions. However, the proposed algorithm outperforms both SOMSA and OFDMA in terms of 


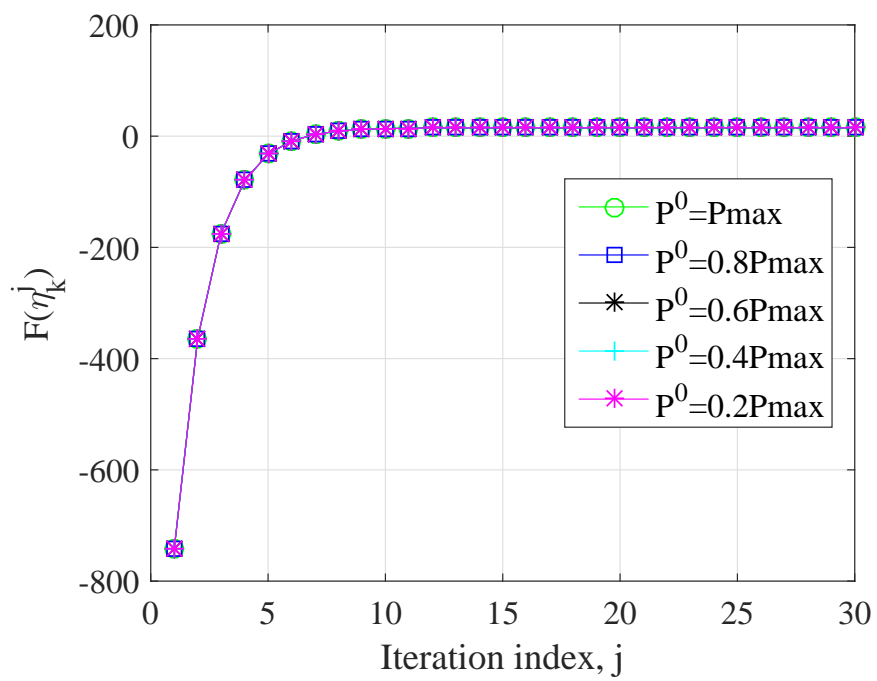

Fig. 3: The convergence of the proposed algorithm 3, the bisection method for maximizing the minimum user's EE (Max-Min EE)

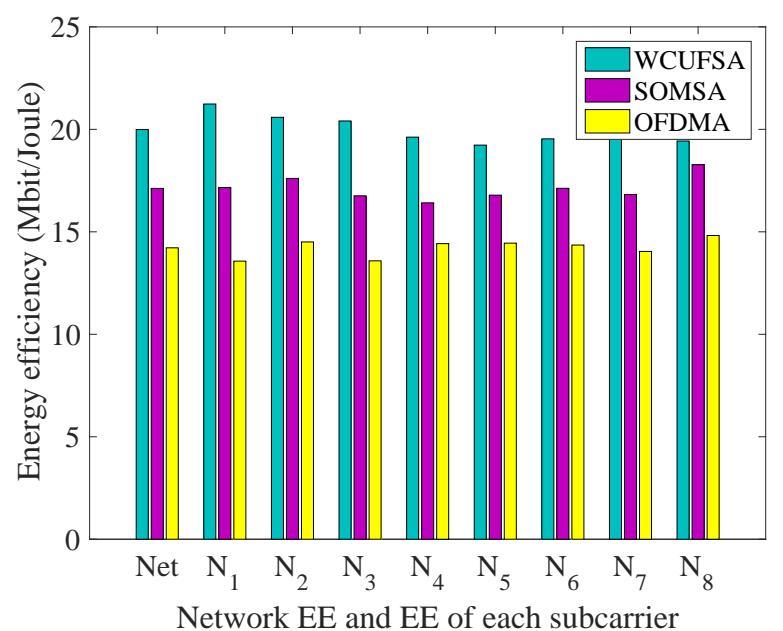

Fig. 4: The EE performance of the network and each subcarrier of three schemes.

EE as well as fairness among users. In Fig. 5, we further compare the EE performance to evaluate the worst link, the best link, as well as the performance of the network's EE among the comparable benchmark schemes in terms of EE. It is observed that there is a remarkable difference in the EE among the best link and the worst link in all considered scenarios. However, the EE of NOMA-SCP is well balanced with slightly reduced from network EE as compared to NOMA-DC and NOMAEQ schemes in a system with 8 subchannels. Fig. 6 shows the achieved data rate of the four schemes against number of users. As it can be seen in Fig. 6, all NOMA schemes are superior to OFDMA schemes in terms of data rate due to the multiplexing gains in NOMA system. Moreover, it also noted that the performance of NOMA-SCP outperforms that of NOMA-DC and NOMA-EQ. As it can be observed from Fig. 6 , the data rate of the proposed NOMA-SCP scheme is $6.30 \%$ more than that of NOMA-DC in a system with 8 users and

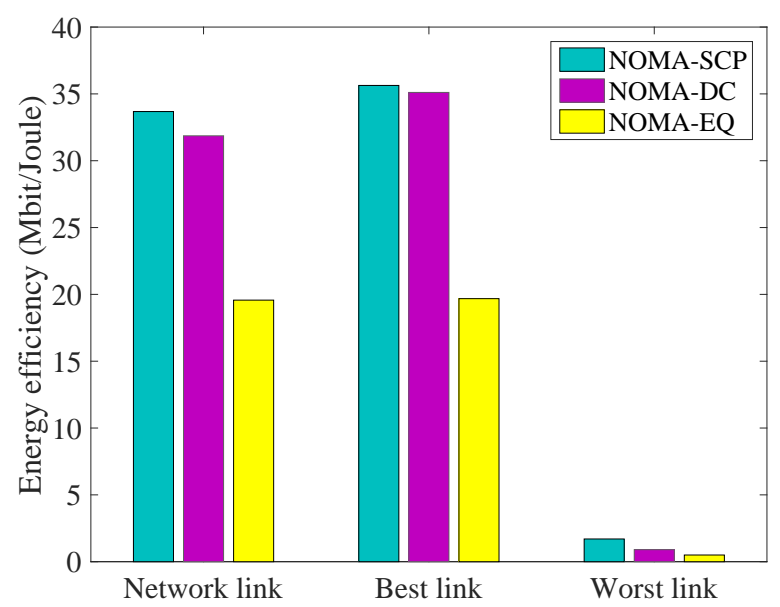

Fig. 5: Comparisons of the EE of the network, the best link, and the worst link among the proposed NOMA-SCP, NOMADC, and NOMA-EQ schemes.

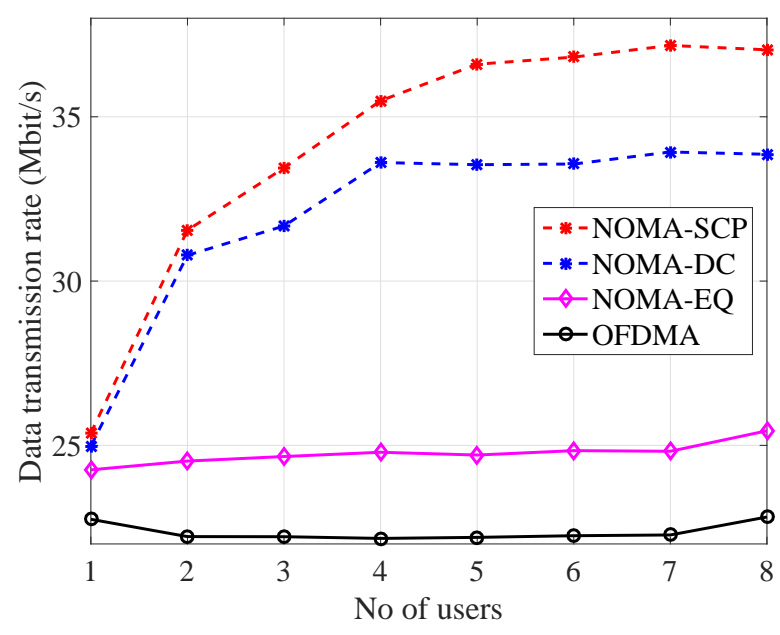

Fig. 6: Data transmission versus number of users

followed by $28.01 \%$ and $35.12 \%$ more than that of NOMAEQ and OFDMA scheme, respectively. Therefore, NOMASCP can achieve a better data rate transmission performance than that of all comparable schemes. Fig. 7 presents the simulation results for the data transmission performance of different power allocation schemes against transmitted power with the same constraints of Fig. 6. Thus, our proposed power allocation scheme through SCP achieves better performance than the benchmark power allocation scheme.

Fig. 8 presents the simulation results of the EE against the number of $K$ users for different power allocation schemes. We set the precision accuracy as $\epsilon=0.001$. In the proposed scheme, the achievable EE initially increases fast as the number of users increases and with slow growth rate afterwards. This is due to the multiuser diversity gain by the NOMA system. From Fig. 8, it is shown that the performance of all NOMA schemes are much better than the OFDMA due to the multiplexing gains when NOMA is used. Moreover, it 


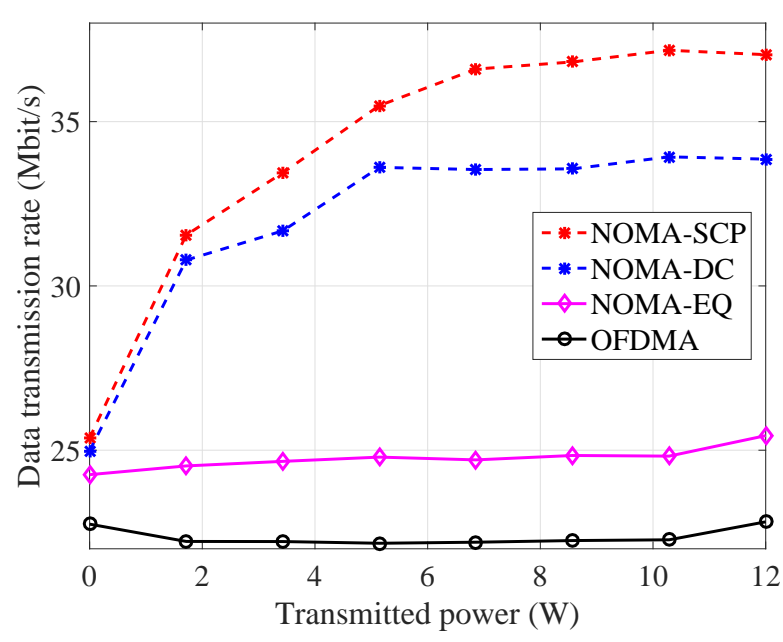

Fig. 7: Data transmission versus transmitted power

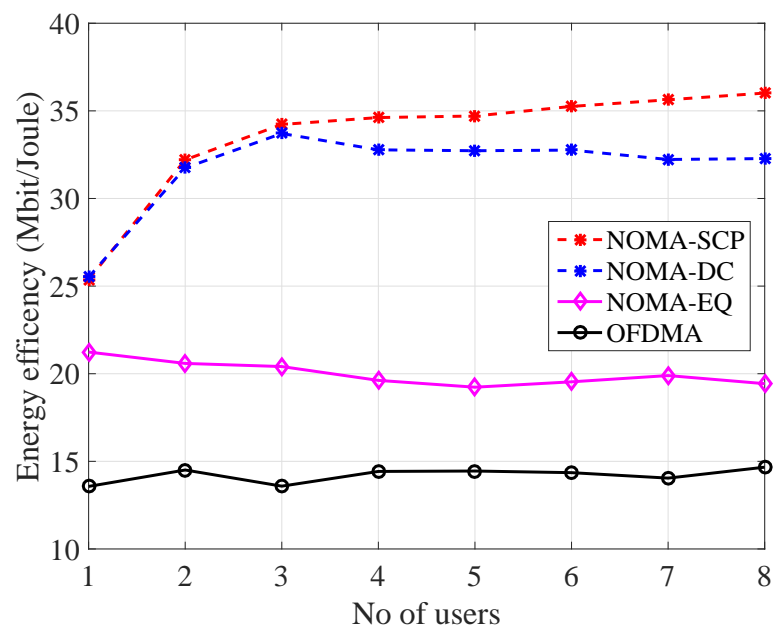

Fig. 8: Energy efficiency versus number of users

also noted that NOMA-SCP outperforms both NOMA-DC and NOMA-EQ in terms of EE. For example, when the number of user is 8 , the EE of NOMA-SCP is $59.21 \%$ more than that of OFDMA scheme. The main reason is that NOMA can support more users in a single subchannel while OFDMA can only support a single user per sub channel. As a result, the BS can not fully utilize spectrum resources as the case of OFDMA system. We also notice that NOMA-SCP improves the EE about $10.38 \%$ compared to NOMA-DC. Fig. 9 demonstrates the EE (i.e., $\eta^{*}$ ) performance versus BS power when fixed circuit power $\mathrm{Pc}=1 \mathrm{~W}$ and the BS power ranges from $1 \mathrm{~W}$ to $12 \mathrm{~W}$. It can be seen that the EE initially increases fast with respect to BS transmitted power and converges with slow growth, due to the total power constraints. This is because when BS power is relatively low, the optimal transmit power selection strategy uses all the available power at the BS. However, when total BS power is large enough, the transmit power selection strategy is limited to $\mathrm{P}^{*}$ regardless of total BS power. From Fig. 9, it is clearly shown that NOMA-SCP can achieve higher EE than NOMA-DC, NOMA-EQ and OFDMA

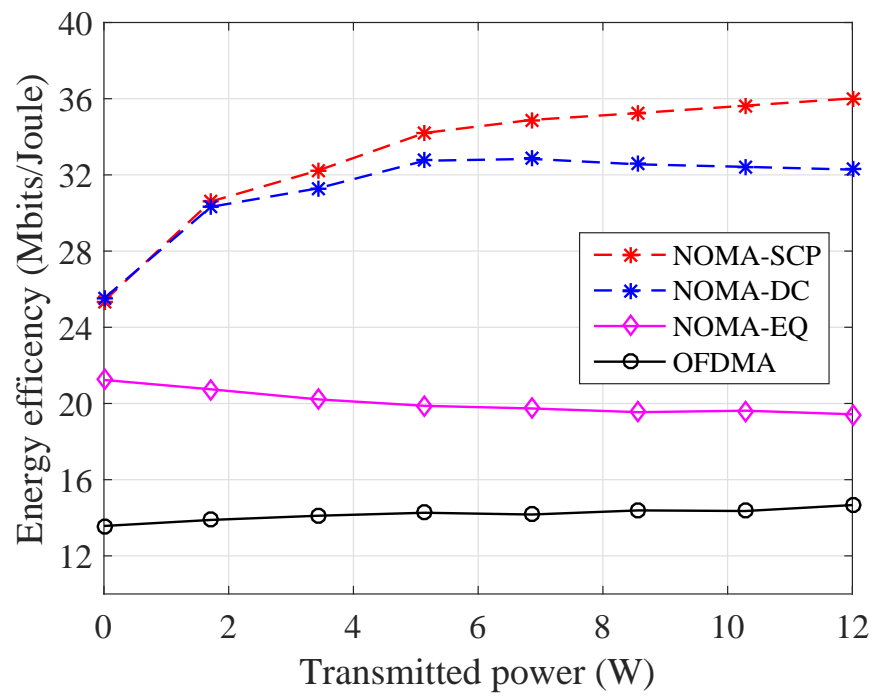

Fig. 9: Energy efficiency versus transmitted power

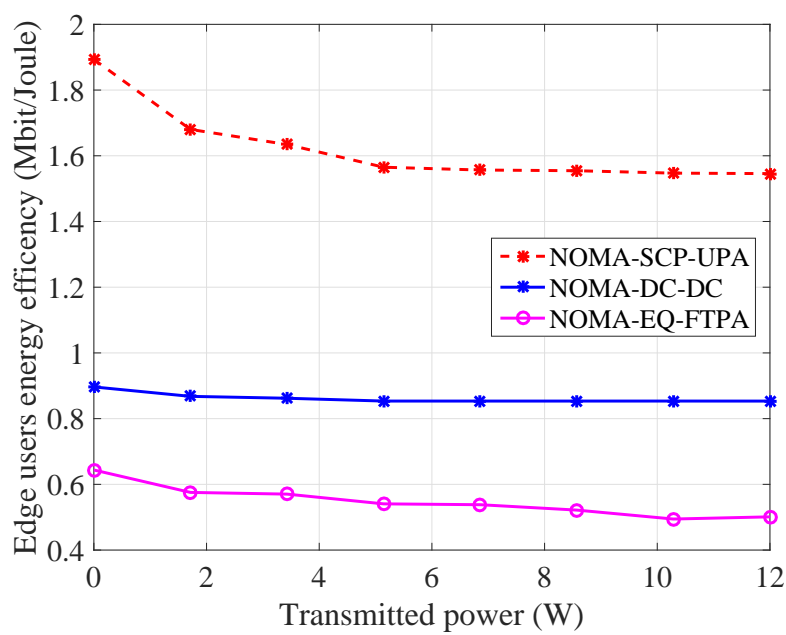

Fig. 10: Edge users EE versus transmitted power

schemes.

In Fig. 10, the effectiveness of different power allocation schemes for multiplexed users is evaluated. Thus, we compare the proposed NOMA-SCP-UPA ${ }^{1}$ scheme with NOMA-DCDC and NOMA-EQ-FTPA, which is widely adopted in NOMA system for power allocation to users in the same subchannel [31], [19]. From Fig. 10, we can clearly see that by using NOMA-SCP-UPA scheme higher EE is achieved. Therefore, the proposed NOMA-SCP-UPA scheme outperforms both NOMA-DC-DC ${ }^{2}$ and NOMA-EQ-FTPA ${ }^{3}$ for edge users in terms of EE. This clearly indicates the effectiveness of the proposed algorithm.

${ }^{1}$ NOMA-SCP-UPA uses SCP approach to allocate power among different subchannels and the bisection search method to assign power between users grouped at the same subchannel.

${ }^{2}$ NOMA-DC-DC uses DC programming techniques to allocate power across subchannels as well as to determine the power allocation factor to allocate power between users grouped at the same subchannel.

${ }^{3}$ NOMA-EQ-FTPA uses equal power allocation across subchannels and FTPA to determine the power allocation factor between users on the same subchannel. 


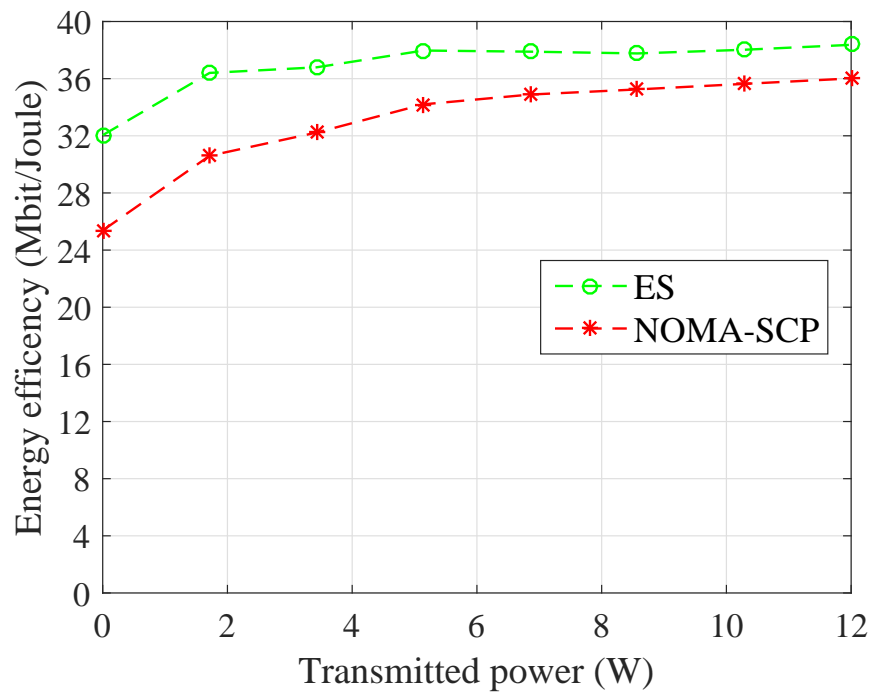

Fig. 11: Energy efficiency versus transmitted power

In order to get further insight on the performance of the proposed scheme, in Fig. 11, we compare the proposed scheme with the optimal solution through exhaustive search (i.e., ES) in terms of EE. It can be observed that the EE increases with the transmit power. It is also noticed that the proposed algorithm is capable of approaching the results of the exhaustive search. Recalling that the complexity of the proposed algorithm is much lower than the one of the exhaustive search, it is concluded that the proposed scheme achieves a good balance between complexity and performance.

\section{CONCLUSions}

In this paper, we have investigated the downlink of MCNOMA system where a single base station transmits a block of messages to multiple users. The focus has been on the maximization of the user with the lowest performance in terms of individual EE by optimizing subcarrier and power allocation. Since the optimization problem was non-convex, we formulated the subcarrier assignment and power allocation as a two stage-problem to reduce computational complexity. Then, a greedy subcarrier assignment scheme to assign two users on each subcarrier was proposed. Furthermore, for the power allocation, we transformed the non-convex problem into a simpler subtractive form using a fractional programming property. Thus, a suboptimal power allocation through the subchannels was obtained by iteratively solving the convex sub-problem using sequential convex programming. The provided simulation results have shown that the proposed resource optimization method achieves fast convergence and guarantees fairness. Consequently, the proposed resource allocation method is particularly promising, since remarkable gains are achieved compared to existing techniques, while it remains appropriate for the practical case.

\section{APPENDIX A}

PROOF OF THEOREM 1:

In complexity theory, to show a decision problem is NPhard, we usually follow three steps [48] 1) choose a suit- able known NP-complete decision problem A; 2) construct a polynomial time transformation from any instance of $\mathrm{A}$ to an instance of the required problem; 3) prove the two instances have the same objective value under the transformation. In the following section, we show that problems (14) is NP-hard.

Proof: The proof can be done into two cases for which $q_{k, n}=1$ and $q_{k, n}>1$.

1) When $q_{k, n}=1$, (14) corresponds to an EE maximization problem with respect to joint subcarrier and power allocation for the conventional OFDMA system, which has been proved to be NP-hard in [47].

2) When $q_{k, n}>1$, we prove that the problem is NP-hard even with known power allocation coefficients. In the following, we construct an instance of problem (14) with known power allocation coefficients. First, we will associate an instance of problem (14) as an equivalent to the Multiple Choice Knapsack problem (MCKP) problem, which is a well known NP-hard problem. We then consider an instance with $q_{k, n}=2$. Thus, we prove a simplified version of the joint subcarrier and power allocation problem is reducible to the knapsack problem which is a well-known NP-hard problem.

Definition 1: Multiple Choice Knapsack problem (MCKP) [48]

Let's assume that there are $N_{1}, N_{2}, \cdots, N_{S}$ classes with each class $i$ containing $n_{i}$ items to be packed in a knapsack with capacity, P. Each item $j \in N_{i}$ has a profit $U_{i, j}$ and a weight $P_{i, j}$ and the problem is to assign some items to each class such that the profit is maximized without having the total weight exceeds P. It is generally considered that the profits, weights and the knapsack capacities take non-negative values.

Thus, we next show that problem in (14) is reduced to MCKP problem. Without loss of generality, we assume that each subcarrier is a knapsack and each item in the knapsack resembles a user to be packed in a knapsack of capacity, $K_{n}$. The profit of each item in the knapsack is the corresponding utility-function is $U_{i, j}$ and the required resource (weight) is $p_{i, j}$, while the Problem in (14) aims at choosing exactly $K_{n}$ users (i.e., items) for each subcarrier (i.e., class) to maximize the EE, subject to the transmit power constraint, $P_{n}$. The EE maximization problem in (14) can be written in the following form:

$$
\begin{array}{ll}
\max _{Q, P} \min _{k=1, \cdots, K} & E_{\eta}(Q, P)=\frac{R_{k, n}(Q, P)}{P_{k, n}^{T}(Q, P)} \\
\text { s.t. } & \mathrm{C}_{3}: \sum_{k=1}^{K_{n}} q_{k, n} P_{k, n} \leq P_{n}, \quad \forall k \in K, \\
& \mathrm{C}_{4}: \sum_{k=1}^{K} q_{k, n} \leq K_{n}, \quad \forall n \in N, \\
& \mathrm{C}_{6}: q_{k, n} \in\{0,1\}, \quad \forall k, n,
\end{array}
$$

Thus, (32) is NP-hard because it is categorized as a MCKP which is a generalization of the ordinary knapsack problem. Thus, as (32) is a special case of problem 
(14), the general optimization problem (14) is an NP-hard problem.

\section{APPENDIX B \\ PROOF OF THEOREM 2:}

Proof: Without loss of generality, we assume that $R_{k}(P) \geq 0$ and $P_{k}(P) \geq 0$, where $P$ and $P^{*}$ denote any feasible power allocation and optimal power allocation policy, respectively, in (14). We also define $e_{k}^{*}$ as the optimal EE for the original objective function in (14). Then, the EE is given by

$$
\max _{\mathcal{P} \in D} \min _{\mathcal{K}} \eta=\frac{R_{k}(P)}{P_{k}(P)}
$$

The equivalent parametric problem related to (33) is

$$
\max _{\mathcal{P}} \min _{\mathcal{K}}\left\{R_{k}(P)-\eta P_{k}(P)\right\}, \forall P \in D .
$$

The following Lemma 1 is introduced to shows the relation between (33) and (34).

Lemma 1: if $P^{*}$ is the optimal solution of (33) with corresponding parameter introduced by $\eta^{*}=\frac{R_{k}\left(P^{*}\right)}{P_{k}\left(P^{*}\right)}$, then $P^{*}$ is also the optimal solution of (33).

Since $P^{*}$ maximizes $\left\{R_{k}(P)-e_{k}^{*} P_{k}(P)\right\}, \forall P \in D$, we have

$$
R_{k}(P)-e_{k}^{*} P_{k}\left(P^{*}\right) \leq R_{k}\left(P^{*}\right)-\eta_{k}^{*} P_{k}\left(P^{*}\right), \forall P \in D .
$$

From the definition of $\eta^{*}$, we have

$$
\left\{R_{k}\left(P^{*}\right)-\eta^{*} P_{k}\left(P^{*}\right)\right\}, \forall P \in D .
$$

Combining (36) and (35), we obtain

$$
\left\{R_{k}(P)-\eta P_{k}^{*}(P)\right\} \leq\left\{R_{k}\left(P^{*}\right)-\eta P_{k}^{*}\left(P^{*}\right)\right\}=0 .
$$

From this

$$
R_{k}(P)-\eta P_{k}\left(P^{*}\right) \leq 0 \text { or } \eta^{*} \geq \frac{R_{k}(P)}{P_{k}(P)}
$$

This indicates that

$$
\eta^{*}=\frac{R_{k}(P)}{P_{k}(P)}, \text { is the maximum of } \frac{R_{k}(P)}{P_{k}(P)}, \forall P \in D \text {. }
$$

In other words $P^{*}$ is the optimal solution of (31). Therefore, the optimal resource allocation for the equivalent objective function is also the optimal resource allocation for the original objective function. This completes the proof.

\section{APPENDIX C}

\section{ProOF OF THEOREM 3:} which

Proof: Let's start with an initial interval $\left[\eta_{\min }, \eta_{\max }\right]$, for

$$
\eta=\frac{\left(\eta_{\min }+\eta_{\max }\right)}{2} \text { and } d=F\left(\eta_{\min }\right) \cdot F\left(\eta_{\max }\right) .
$$

- If $d<0$, let $\eta_{\max }=\eta$ and $\eta_{\min }=\eta_{\min }$.

- If $d>0$, let $\eta_{\min }=\eta$ and $\eta_{\max }=\eta_{\max }$.

- If $d=0$, then $\eta$ becomes the solution with the required accuracy, $\epsilon$.

For either of the two cases, the new interval is one half of the width of the original. This new interval is reformed as $\left[\eta_{\min }, \eta_{\max }\right]$ and the procedure is repeated again. Over the $j$-th iterations, it follows that

- The first interval is $\left[\eta_{\min }^{0}, \eta_{\max }^{0}\right]$ and $\eta^{0}=\frac{\left(\eta_{\min }^{0}+\eta_{\max }^{0}\right)}{2}$

- The Second interval is $\left[\eta_{\min }^{1}, \eta_{\max }^{1}\right]$ and $\eta^{1}=\frac{\left(\eta_{\min }^{1}+\eta_{\max }^{1}\right)}{2}$

- The $j$-th interval is $\left[\eta_{\min }^{j}, \eta_{\max }^{j}\right]$ and $\eta^{j}=\frac{\left(\eta_{\min }^{j}+\eta_{\max }^{j}\right)}{2}$ where $\eta_{\min }^{j}=\eta^{j-1}$ and $\eta_{\max }^{j}=\eta_{\max }^{j-1}$ or $\eta_{\min }^{j}=\eta_{\min }^{j-1}$ and $\eta_{\max }^{j}=\eta^{j-1}$. From this we can observe that

- The sequence $\left\{\eta_{\min }^{j}\right\}_{j=0}^{j=\infty}$ is increasing sequence and bounded above by $\eta_{\max }$.

- The sequence $\left\{\eta_{\max }^{j}\right\}_{j=0}^{j=\infty}$ is decreasing sequence and bounded below by $\eta_{\min }$.

- and the approximated sequence of $\eta^{j}$ 's generated by the bisection is found on $\eta_{\min }^{j} \leq \eta^{j} \leq \eta_{\max }^{j}$, for all $j$. Moreover, the function $F(\eta)$ is strictly decreasing in $\eta$ [36], [37]. In addition, $F(\eta)$ is negative for $\eta \rightarrow+\infty$ and positive for $\eta \rightarrow-\infty$. This satisfied $F\left(\eta_{j}^{\min }\right) \cdot F\left(\eta_{j}^{\max }\right)<0$

Furthermore, let us define the approximation at $\eta^{j}$ after the $j$-th iteration as the midpoint

$$
\eta^{j}=\frac{\left(\eta_{\min }^{j}+\eta_{\max }^{j}\right)}{2}
$$

Since the actual solution $F\left(\eta^{*}\right)=0$ satisfies $\eta \in \frac{\eta_{\max }^{j}-\eta_{\min }^{j}}{2}$, we have

$$
\left|\eta^{j}-\eta^{*}\right|<\frac{1}{2}\left|\frac{\eta_{\max }^{j}-\eta_{\min }^{j}}{2}\right|
$$

Since the length of the current search interval gets divided in half in each iteration, we have

$$
\left|\epsilon^{j}\right|=\left|\eta^{j}-\eta^{*}\right| \leq\left(\frac{1}{2}\right)^{j}\left|\frac{\eta_{\max }^{j}-\eta_{\min }^{j}}{2}\right| .
$$

From this, we have $\lim _{j \rightarrow \infty} e^{j}=0$. For $\lim _{j \rightarrow \infty} \frac{1}{2^{j}}=0$, we obtain $\eta^{j}=\eta^{*}$, which proves the global convergence of the bisection method. We interpret this behavior as linear convergence.

Moreover, let the $\epsilon$ be the relative accuracy of the root, then to estimate the number of iteration $j$ to achieve the accuracy is given by

$$
\frac{\left|\eta^{j}-\eta^{*}\right|}{\left|\eta^{*}\right|} \leq \epsilon
$$

Let's assume that the root lies in $\left[\eta_{\min }, \eta_{\max }\right]$ where $\eta_{\max }>$ $\eta_{\min }>0$. Clearly, $\left|\eta^{*}\right| \geq \eta^{\text {min }}$ and hence the above relation is true if

$$
\frac{\left|\eta^{j}-\eta^{*}\right|}{\eta^{*}} \leq \epsilon
$$

which is true if

$$
\frac{\eta_{\max }-\eta_{\min }}{\left(2^{j+1}\right) \eta *} \leq \epsilon
$$

Solving this we can find the minimum number of iterations needed to obtain the desired accuracy. Now, it can be derived that

$$
\left|e^{j+1}\right|=\left|\eta^{j+1}-\eta^{*}\right| \leq \frac{1}{2}\left(\eta_{\max }^{j+1}-\eta_{\min }^{j+1}\right)=\frac{1}{2}\left(\frac{\eta_{\max }-\eta_{\min }}{2}\right)
$$


and

$$
\left|e^{j}\right|=\left|\eta^{j}-\eta^{*}\right| \leq \frac{1}{2}\left(\eta_{\max }^{j}-\eta_{\min }^{j}\right) .
$$

Thus, we find $\left|e_{j+1}\right| \approx \frac{1}{2}\left|e_{j}\right|$.

Therefore, the proposed bisection method in order to determine $\eta^{*}$ converges linearly. This completes the proof.

\section{APPENDIX D \\ PROOF OF THEOREM 4:}

As $P^{t}$ is feasible to (31), it follows that

$$
\begin{array}{r}
E^{t}=\min _{k}\left(f_{k}\left(P^{t+1}\right)-g_{k}\left(P^{t+1}\right) \geq \min _{k}\left(f_{k}(P)-\left[g_{k}\left(P^{t}\right)\right.\right.\right. \\
\left.+\nabla g_{k}^{T}\left(P^{t}\right)\left(P^{t+1}-P^{t}\right)\right] \geq \min _{k}\left(f_{k}\left(P^{t}\right)-g_{k}\left(P^{t}\right)\right)=E^{t+1}
\end{array}
$$

The next solution $P^{t+1}$ is always better than the previous solution $P^{t}$. That is $\min \left(f_{k}\left(P^{t}\right)-g_{k}\left(P^{t}\right)\right)$ monotonically decreases when the iteration $\mathrm{t}$ increases. With successive iterations of the algorithm, the value of $E^{(t)}=\min \left(f_{k}\left(P^{t}\right)-\right.$ $\left.g_{k}\left(P^{t}\right)\right)$ decreases. Moreover, for every $E^{(t)}$ the power vector $\mathrm{P}$ that maximize $f_{k}(P)-\left[g_{k}\left(P^{t}\right)+\nabla g_{k}^{T}\left(P^{t}\right)\left(P-P^{t}\right)\right]$ is found. Thus, iteration process terminates after a finite iteration at $\min \left(f_{k}\left(P^{t}\right)-g_{k}\left(P^{t}\right)\right) \leq \epsilon$ (no solution progress) with some threshold $\epsilon \geq 0$. Hence, the iterative power control algorithm converges in a finite step. Furthermore, since the constraint set is compact, by Cauchy Theorem the sequence $P^{t}$ of improved solution always converges [42]. From this, we can conclude that Algorithm 3 is guaranteed to converge.

b) Proof of optimal transmit power converges to a stationary point Consider Proof of algorithm convergence, we now prove problem (31) in algorithm 3 for optimal transmit power converges to a stationary point under an additional assumption $f_{k}(P)$ and $g_{k}(P)$ defined in $f_{k}(P)-g_{k}(P)$ are continuous and differentiable over a given constraint sets. Since $-g_{k}(P)$ is approximate by its convex function as

$$
g_{k}\left(P^{t}\right)+\nabla g_{k}^{T}\left(P^{t}\right)\left(P-P^{t}\right)
$$

The objective function is rewritten as

$$
Q_{k}(P)=f_{k}\left(P^{t}\right)-\left[g_{k}\left(P^{t}\right)+\nabla g_{k}^{T}\left(P^{t}\right)\left(P-P^{t}\right)\right]
$$

In the limit all inequalities in (49) become equality. In other words, $P^{t}$ and $P^{t+1}$ are optimal point of the objective function over the defined constraint sets [35]. Hence, $P^{t}=P^{t+1}$ and

$$
P^{t+1}=\arg \max _{P \in\left\{C^{\prime} 1, C 2, C 4\right\}} \min _{\mathcal{K}} Q_{k}(P)
$$

Furthermore, according to optimality condition [35], we have

$$
\min _{\mathcal{K}} \nabla Q_{k}^{T}\left(P^{t}\right)\left(P-P^{t}\right)=\min _{\mathcal{K}}\left\{\nabla Q_{k}\left(P^{t+1}\right)\left(P-P^{t+1}\right)\right\} \leq 0
$$

which can be equivalent to [40]

$$
\min _{\mathcal{K}}\left\{\nabla f_{k}\left(P^{t}\right)+\nabla g_{k}^{T}\left(P^{t}\right)\left(P-P^{t}\right)\right\} \leq 0 .
$$

Thus, $P^{t}$ is the stationary point to (31) i.e. (20). This completes the proof.

\section{REFERENCES}

\section{REFERENCES}

[1] L. Dai, B. Wang, Y. Yuan, S. Han, C. 1. I, and Z. Wang, "Non-orthogonal multiple access for 5G: Solutions, challenges, opportunities, and future research trends," IEEE Commun. Mag., vol. 53, no. 9, pp. 74-81, Sept. 2015.

[2] Z. Wei, J. Yuan, D. W. K. Ng, M. Elkashlan, and Z. Ding, "A survey of downlink non-orthogonal multiple access for $5 \mathrm{G}$ wireless communication networks," ZTE Communications, vol. 14. 17-25, Oct. 2016.

[3] M. R. Hojeij, J. Farah, C. A. Nour, and C. Douillard, "Resource allocation in downlink non-orthogonal multiple access (NOMA) for future radio access," in Proc. IEEE Veh. Technol. Conf., Dresden, Germany, May. 2015, pp. 1-6.

[4] S. Zhang, B. Di, L. Song, and Y. Li, "Radio resource allocation for non-orthogonal multiple access (NOMA) relay network using matching game," in Proc. Int. Conf. Commun., KUL, Malaysia, May. 2016, pp. $1-6$

[5] Z. Q. Al-Abbasi and D. K. C. So, "Resource allocation in nonOrthogonal and hybrid multiple access system with proportional rate constraint," IEEE Trans.Wireless Commun., vol. 16, no. 10, Oct, 2017.

[6] Z. Wei, D. W. K. Ng, and J. Yuan, "Power-efficient resource allocation for MC-NOMA with statistical channel state information," in Proc. IEEE Global Commun. Conf., Dec. 2016, pp. 1-7.

[7] Z. Wei, D. W. K.Ng, J.Yuan, and H. Wang, "Optimal resource allocation for power-efficient MC-NOMA with imperfect channel state information," IEEE Trans. Commun, vol. 65, no. 9. Sept. 2017.

[8] J. Shi and L.-L. Yang, "Novel subcarrier-allocation schemes for downlink MC DS-CDMA systems," IEEE Trans. Wireless Commun., vol. 13,no. 10, pp. 5716-5728, Oct. 2014.

[9] Y. Sun, D. W. K. Ng, Z. Ding, and R. Schober, "Optimal joint power and subcarrier allocation for MC-NOMA systems," in Proc.IEEE Global Commun. Conf., Washington, DC, USA, Dec. 2016, pp. 1-6.

[10] J. Wang, Q. Peng, Y. Huang, H.-M. Wang, and X. You, "Convexity of weighted sum rate maximization in NOMA systems," IEEE Commun. Lett., vol. 24, no. 9, pp. 1323-1326, Sept. 2017.

[11] L. Lei, D. Yuan, C. K. Ho, and S. Sun, "Joint optimization of power and channel allocation with non-orthogonal multiple access for $5 \mathrm{G}$ cellular systems," in Proc. IEEE Globecom, Dec. 2015,pp. 1-6

[12] P. Xu and K. Cumanan, "Optimal power allocation scheme for nonorthogonal multiple access with $\alpha$-fairness," IEEE Journal on Selected Areas in Communication, vol. 35, pp. 2357 - 2369, Oct. 2017.

[13] J. Cui, Z. Ding, and P. Fan, "A novel power allocation scheme under outage constraints in NOMA systems," IEEE Signal Process. Lett.,vol. 23, no. 9, pp. 1226-1230, Sept. 2016.

[14] S. Timotheou and I. Krikidis, "Fairness for non-orthogonal multiple access in 5G systems," IEEE Signal Process. Lett., vol. 22, no. 10, pp. 1647-1651, Oct. 2015.

[15] J. Choi, "Power allocation for max-sum rate and max-min rate proportional fairness in NOMA," IEEE Commun. Lett., vol. 20, no. 10, pp.2055-2058, Oct. 2016.

[16] Y. Li, M. Sheng, C. W.Tan, Y. Zhang, Y. Sun, X. Wang, Y. Shi and J. Li, "Energy-efficient subcarrier assignment and power allocation in OFDMA systems with Max-Min fairness guarantees," IEEE Trans. Commun, vol. 63, no. 9, Sept 2015.

[17] W. Hao, M. Zeng, Z. Chu, and S. Yang, "Energy-efficient power allocation in millimeter wave massive mimo with non-orthogonal multiple access," IEEE Wireless Commun. Lett., vol. PP, no. 99, pp. 782-785, 2017.

[18] Y. Zhang, H. M. Wang, T. X. Zheng, and Q. Yang, "Energy-efficient transmission design in non-orthogonal multiple access," IEEE Trans. Veh. Technol., vol. 66, no. 99, pp. 2852-2857, Sept. 2016.

[19] F. Fang, H. Zhang, J. Cheng, and V. C. M. Leung, "Energy-efficient resource allocation for downlink non-orthogonal multiple access network," IEEE Trans. Commun., vol. 64, no. 9, pp. 3722-3732, May. 2016.

[20] F. Fang, J. Cheng and Z.Ding, "Joint Energy Efficient Subchannel and Power Optimization for a Downlink NOMA Heterogeneous Network," IEEE Trans. on vehicular technolgy, vol. 68, no. 2, Feb. 2019.

[21] K. Higuchi and A. Benjebbour, "Non-orthogonal multiple access (NOMA) with successive interference cancellation for future radio access," IEICE Transactions on Communications, vol. E98.B, no. 3, pp. 403-414, 2015

[22] S. He, Y. Huang, S. Jin, F. Yu, and L. Yang, "Max-min energy efficient beamforming for multicell multiuser joint transmission systems," IEEE Commun. Lett., vol. 17, no. 10, pp. 1956-1959, Oct. 2013. 
[23] Z. Ding, Z. Yang, P. Fan, and H. Poor, "On the performance of nonorthogonal multiple access in $5 \mathrm{G}$ systems with randomly deployed users," IEEE Signal Processing Lett, vol. 21, no. 12, pp. 1501-1505, Dec. 2014.

[24] C. L. Wang, J. Y. Chen, and Y. J. Chen, "Power allocation for a downlink non-orthogonal multiple access system," IEEE Wireless Commun. Lett., vol. 5, no. 5, pp. 532-535, Oct. 2016.

[25] J. Zhu, J. Wang, Y. Huang, S. He, X. You, and L. Yang, "On optimal power allocation for downlink non-orthogonal multiple access systems," IEEE J. Sel. Areas Commun., vol. 35, no. 12, pp. 2744-2757, Dec. 2017.

[26] Y. Yang and M. Pesavento, "A Parallel algorithm for energy Efficiency Maximization in massive MIMO networks," in IEEE Global Commun. Conf. (Globecom), pp. 1-6, 2016

[27] G. Li, J. Yang, X.Liu , Q.Yang and Y. Xin ,"Fairness-aware energyefficient resource allocation for uplink OFDMA networks with statistical QoS requirements," IEEE, conf. pp. 58-62, 2016.

[28] A. Zappone, L. Sanguinetti, G. Bacci, E. A. Jorswieck, and M. Debbah, "A framework for energy-efficient design of 5G technologies," in Proc. IEEE Int. Conf. Commun. (ICC), London, U.K.Jun. 2015, pp. 18451850.

[29] Y. Saito, Y. Kishiyama, A. Benjebbour, T. Nakamura, A. Li, and K. Higuchi, "Non-orthogonal multiple access (NOMA) for cellular future radio access," in Proc. IEEE Vehicular Technology Conference (VTC Spring), Dresden, Germany, Jun. 2013, pp. 1-5.

[30] G. Ye, H. Zhang, H. Liu, J. Cheng, and V. C. M. Leung, "Energy efficient joint user association and power allocation in a two-tier heterogeneous network," in IEEE Global Commun. Conf. (Globecom), 2016, pp. 1-5.

[31] P. Parida and S. S. Das, "Power allocation in OFDM based NOMA systems: A DC programming approach," in IEEE Globecom Workshops (GC Wkshps), Austin, 2014, pp. 1026-1031.

[32] S. Zarandi and M. Rasti, "Energy efficient resource allocation in twotier heterogeneous network with inband full-duplex communications," ICEE, conf. Tehran, Iran, pp. 2072-2077, 2017.

[33] L. Xu, G. Yu, and Y. Jiang, "Energy-efficient resource allocation in single cell OFDMA systems: Multi-objective approach," IEEE Trans. Wireless Commun., vol. 14, no. 10, pp. 5848-5858, Oct. 2015.

[34] Zappone, E.A. Jorswieck, "Energy-efficient resource allocation in future wireless networks by sequential fractional programming," Digital Signal Processing, vol 60, 2017, pp. 324-337.

[35] S. Boyd and L. Vandenberghe, Convex optimization. Cambridge University Press, New York, NY, 2004.

[36] A. Zappone and E. Jorswieck, "Energy efficiency in wireless networks via fractional programming theory," Foundations and Trends in Communications and Information Theory, vol. 11, no. 3-4, pp. 185-396, 2015.

[37] W. Dinkelbach, "On nonlinear fractional programming," Management Science, vol. 13, no. 7, pp. 492-498, Mar. 1967.

[38] M. Cui, B.-J. Hu, H. Chen, and X. Li, "Max-min fair power control algorithm in massive MIMO cognitive radio networks," in Proc. IEEE WCSP, Oct. 2016, pp. 1-5.

[39] M. S. Ali, H. Tabassum, and E. Hossain, "Dynamic user clustering and power allocation for uplink and downlink non-orthogonal multiple access (NOMA) systems," IEEE Access, vol. 4, pp. 6325-6343, Aug.2016.

[40] Y. Li, M. Sheng, X. Wang, Y. Zhang, and J. Wen, "Max-min energy efficient power allocation in interference-limited wireless networks," IEEE Trans. Veh. Technol., vol. 64, no. 9, pp. 4321-4326, Sep. 2015.

[41] M. Naeem, K. Illanko, A. Karmokar, A. Anpalagan, and M. Jaseemuddin, "Optimal power allocation for green cognitive radio: fractional programming approach," IET Comm., vol. 7, no. 12, 2013.

[42] H. Kha, H. Tuan, and H. Nguyen, "Fast global optimal power allocation in wireless networks by local D.C. programming," IEEE Trans. Wireless Commun., vol. 11, no. 2, pp. 510-515, Feb. 2012.

[43] F. Fang ; J. Cheng ; Z. Ding ; H. V. Poor, "Energy Efficient Resource Optimization for a Downlink NOMA Heterogeneous Small-cell Network," IEEE conf., pp. 51-55, 2018.

[44] Y. Li, P. Fan, and N. C. Beaulieu, "Cooperative downlink maxmin energy-efficient precoding for multicell MIMO networks," IEEE Trans.Veh. Technol, vol. 65, no. 11, pp. 9425-9430, Nov. 2016

[45] A. Fehske, G. Fettweis, J. Malmodin, and G. Biczok, "The global footprint of mobile communications: the ecological and economic perspective," IEEE Commun. Mag., vol. 49, no. 8, pp. 55-62, Aug. 2011.

[46] D. T. Ngo, S. Khakurel, and T. Le-Ngoc, "Joint subchannel assignment and power allocation for OFDMA femtocell networks," IEEE Trans.Wireless Commun., vol. 13, no. 1, pp. 342-355, Jan. 2014.

[47] Y. F. Liu and Y. H. Dai, "On the complexity of joint subcarrier and power allocation for multi-user OFDMA systems," IEEE Transactions on Signal Processing, vol. 62, no. 3, pp. 583-596, Feb 2014.
[48] M. R. Garey and D. S. Johnson, Computer and Intractability: A Guide to the Theory of NP-Completeness., WH Freemann, New York, 1979.

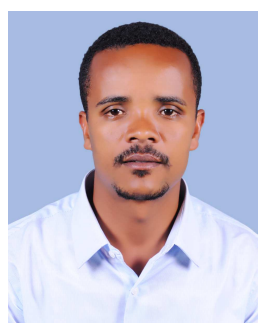

Alemu Jorgi Muhammed (M'19) received the BSc and MSc degrees in Information Technology and Information Science from Addis Ababa University, Ethiopia, in 2005 and 2010, respectively. $\mathrm{He}$ is currently pursuing the $\mathrm{PhD}$ degree in Information and Communication Engineering at the school of Information Science and Technology, SouthWest Jiaotong University, Chengdu, China. His current research interests include 5G network, Energy Efficient communication, Non-Orthogonal multiple acand optimization theory. cess (NOMA), Heterogeneous small cell networks

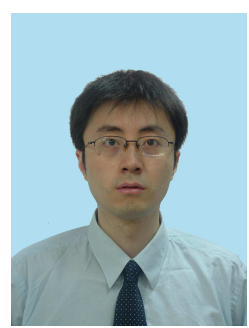

Zheng Ma (M'07) received the B.Sc. and Ph.D. degrees in Communications and Information System from Southwest Jiaotong University in 2000 and 2006, respectively. He was a Visiting Scholar with the University of Leeds, U.K., in 2003. In 2003 and 2005, he was a Visiting Scholar with The Hong Kong University of Science and Technology. From 2008 to 2009, he was a Visiting Research Fellow with the Department of Communication Systems, Lancaster University, U.K. He is currently a Professor with Southwest Jiaotong University, and servers as a Deputy Dean of the School of Information Science and Technology. He has authored over 60 research papers in high quality journals and conferences. His research interests include information theory and coding, signal design and applications, FPGA/DSP implementation, and professional mobile radio. $\mathrm{He}$ is currently the Editor of the IEEE COMMUNICATIONS LETTERS. He is also the Vice Chairman of Information Theory Chapter in the IEEE Chengdu Section.

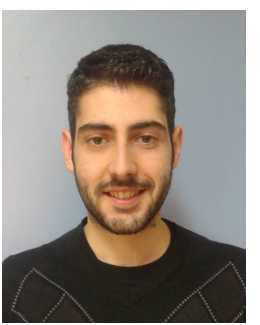

P ANAGIOTIS D. DIAMANTOULAKIS (SM'18) received the Diploma degree (5 years) and the $\mathrm{Ph} . \mathrm{D}$. degree from the Department of Electrical and Computer Engineering, Aristotle University of Thessaloniki (AUTH), Greece, in 2012 and 2017, respectively, where he is currently working as a Postdoctoral Fellow with the Wireless Communications Systems Group (WCSG). During 2018-2019, he has also been Visiting Post-doctoral Researcher in the Key Lab of Information Coding and Transmission at Southwest Jiaotong University, China. His current research interests include resource allocation in wireless communications, optimization theory and applications, game theory, non-orthogonal multiple access, and wireless power transfer. He serves as an Editor for the IEEE WIRELESS COMMUNICATIONS LETTERS and the Elsevier Physical Communications. Also, he was an Exemplary Reviewer of the IEEE COMMUNICATIONS LETTERS in 2014, and the IEEE TRANSACTIONS ON WIRELESS COMMUNICATIONS 2017 (top 3

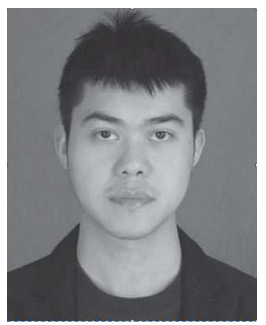

$\mathbf{L i} \mathbf{L i}$ received the Ph.D. degree from Southampton Wireless Group, School of Electronics and Computer Science, University of Southampton, in 2013. After completing his Ph.D. degree, he conducted a research as a Senior Research Assistant at the School of Electronics and Computer Science, University of Southampton, from 2013 to 2014. In 2015, he joined the Provincial Key Laboratory of Information Cod ing and Transmission, Southwest Jiaotong University, Chengdu, China, as an Associate Professor. His research interests include channel coding, iterative detection, noncoherent transmission technologies, cooperative communica tions, network coding, and non-orthogonal multiple access technologies. $\mathrm{He}$ currently serves as an Editor for IEEE COMMUNICATIONS LETTERS. 


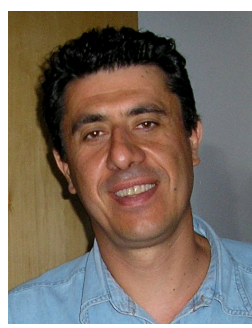

George K. Karagiannidis (M'96-SM'03-F'14) received the University Diploma (5years) and $\mathrm{PhD}$ degree, both in electrical and computer engineering from the University of Patras, in 1987 and 1999, respectively. From 2000 to 2004, he was a Senior Researcher at the Institute for Space Applications and Remote Sensing, National Observatory of Athens, Greece. In June 2004, he joined the faculty of Aristotle University of Thessaloniki, Greece where he is currently Professor in the Electrical \& Computer Engineering Dept. and Director of Digital Telecommunications Systems and Networks Laboratory.

$\mathrm{He}$ is also Honorary Professor at South West Jiaotong University, Chengdu, China. His research interests are in the broad area of Digital Communications Systems and Signal processing, with emphasis on Wireless Communications, Optical Wireless Communications, Wireless Power Transfer and Applications, Molecular and Nanoscale Communications, Stochastic Processes in Biology and Wireless Security.

$\mathrm{He}$ is the author or co-author of more than 450 technical papers published in scientific journals and presented at international conferences. He is also author of the Greek edition of a book on "Telecommunications Systems" and co-author of the book "Advanced Optical Wireless Communications Systems", Cambridge Publications, 2012. Dr. Karagiannidis has been involved as General Chair, Technical Program Chair and member of Technical Program Committees in several IEEE and non-IEEE conferences. In the past, he was Editor in IEEE Transactions on Communications, Senior Editor of IEEE Communications Letters, Editor of the EURASIP Journal of Wireless Communications \& Networks and several times Guest Editor in IEEE Selected Areas in Communications. From 2012 to 2015 he was the Editor-in Chief of IEEE Communications Letters. Dr. Karagiannidis is IEEE Fellow and one of the highly-cited authors across all areas of Electrical Engineering, recognized as 2015, 2016 and 2017 Thomson-Reuters Highly-Cited Researcher. 